\title{
Fault Detection for Uncertain LPV Systems Using Probabilistic Set-membership Parity Relation
}

\author{
Yiming Wan ${ }^{\mathrm{a}, \mathrm{b}, *}$, Vicenç Puig ${ }^{\mathrm{c}}$, Carlos Ocampo-Martinez ${ }^{\mathrm{c}}$, Ye Wang ${ }^{\mathrm{d}}$, Eranda \\ Harinath $^{\mathrm{e}}$, Richard D. Braatz ${ }^{\mathrm{e}}$ \\ ${ }^{a}$ School of Artificial Intelligence and Automation, Huazhong University of Science and \\ Technology, Wuhan 430074, China \\ ${ }^{b}$ Key Laboratory of Image Processing and Intelligent Control (Huazhong University of \\ Science and Technology), Ministry of Education China \\ ${ }^{c}$ Universitat Politècnica de Catalunya, Institut de Robòtica i Informàtica Industrial (CSIC, \\ UPC), C/. Llorens i Artigas 4-6, 08028 Barcelona, Spain \\ ${ }^{d}$ College of Automation, Harbin Engineering University, No.145 Nantong Street, Nangang \\ District, 150001 Harbin, China \\ ${ }^{e}$ Massachusetts Institute of Technology, Cambridge, MA 02139, USA
}

\begin{abstract}
This paper considers fault detection of uncertain linear parameter varying systems that have polynomial dependence on parametric uncertainties. A conventional set-membership (SM) approach is able to ensure zero false alarm rate (FAR) by using conservative threshold sets, but usually results in a high missed detection rate (MDR) due to equally treating all uncertainty realizations without distinguishing between high and low probability of occurrence. To address this limitation, a probabilistic SM parity relation approach is proposed to exploit probabilistic information on the parametric uncertainties, which results in a reduced MDR by admitting an acceptable FAR. The parity relation is first polynomially parameterized with respect to uncertain parameters. Then, Gaussian mixtures are adopted to efficiently compute uncertainty propagation from stochastic uncertainties to the residual distribution. To achieve an acceptable FAR, a non-convex confidence set of residuals - represented by a union of ellipsoids - is determined for the consistency test. The effectiveness of the proposed approach is illustrated using a continuous stirred tank reactor example including performance comparisons with a deterministic zonotope-based method.
\end{abstract}

Keywords: Fault detection, linear parameter varying systems, probabilistic parametric uncertainties, parity relation, set membership approach. 


\section{Introduction}

The inherent nonlinearity of practical safety-critical systems has motivated recent research interest in nonlinear fault detection (FD) 1, 2, 3. Linear parameter varying (LPV) models effectively represent a wide range of nonlinear

5 systems while preserving a (quasi-)linear structure, which has motivated their use in the design of nonlinear FD methods 1, 4. As in other FD methods, a key challenge for an LPV FD algorithm is to ensure robustness to inevitable model uncertainties to achieve a low false alarm rate (FAR), while simultaneously obtaining a low missed detection rate (MDR).

10 The existing robust LPV methods can be classified into two main categories. The first category relies on an LPV observer or a parity relation to generate residuals or fault estimates, and uses a norm-based threshold to indicate the occurrence of faults [5, 6, 7, 8, 9, 10, 11]. On the other hand, the second category of robust methods, known as set membership (SM), performs a more flexible

15 set-based consistency test via a set-valued observer [12, 13, 14, or an SM parity relation [15, 16]. For tractable online computation in set-theoretic uncertainty propagation, convex outer approximations using intervals, ellipsoids, or zonotopes are often adopted, which compromises the MDR. A parity relation is a receding horizon input-output (I/O) model decoupled from the unknown initial

20 states by using a so-called parity matrix or vector [11, 17]. To effectively address multiplicative uncertainties, the parity relation approach has been revised in the SM framework [15, 16]. The derived SM parity relation is parameterized by uncertain parameters, in contrast to a single parity relation as used in [11, 17.

The above robust approaches are developed in the deterministic setting, and aim at ensuring zero false alarms even in the worst case. The resulting MDR is high with such methods, because conservative detection thresholds or sets must be adopted to account for the worst case that rarely occurs. In order to reduce this conservatism, probabilistic robust approaches have been recently proposed

so that exploit the richer information represented by probability distribution of model uncertainties. One line of research formulates a probabilistic relaxation for the norm-based threshold computation [18, 19, 20, 21, while the other line employs probabilistic set approximation for the set-based consistency test [22]. The latter approach achieves better FD performance by adopting less conserva-

35 tive polynomial level sets, but requires solving chance-constrained optimization online via a computationally expensive randomized algorithm. To address the presence of both bounded deterministic parametric uncertainties and additive Gaussian noises, a zonotopic Kalman filter is proposed in [23, 24] by merging $\mathrm{SM}$ and probabilistic paradigms.

40 This paper investigates the FD problem for uncertain LPV systems with polynomial dependence on probabilistic parametric uncertainties. A probabilistic SM parity relation approach is proposed to reduce the MDR while ensuring an acceptable FAR. The polynomially parametrized parity relation is first derived, and then restructured as a linear time-varying (LTV) transformation of an 45 uncertainty vector whose elements are polynomials of uncertain parameters and 
stochastic noises. Considering non-Gaussian uncertainties, Gaussian mixtures (GMs) are adopted to efficiently compute stochastic uncertainty propagation from the above uncertainty vector to the residual distribution. The confidence set of the residual GM distribution is used in the consistency test to achieve an acceptable FAR. Such a confidence set is represented by a union of ellipsoids, which is a less conservative approximation of a non-convex region than the convex approximation used in [15, 16. The proposed approach is a nontrivial extension of the authors' previous work for uncertain linear time-invariant systems in [25, 26, to LPV systems including both parametric uncertainties and 55 imprecise scheduling parameters.

The paper is organized as follows. Sections 2 and 3 state the probabilistic FD problem and the main idea, respectively. Section 4 constructs a polynomial parity matrix. The residual uncertainty quantification for the consistency test is discussed in Section 5. The simulation results using a continuous stirred tank reactor example are presented in Section 6, and the main conclusions are drawn in Section 7.

\section{Problem statement}

Consider the fault-free uncertain discrete-time LPV system

$$
\begin{aligned}
x_{k+1} & =A\left(\tilde{\theta}, \tilde{\rho}_{k}\right) x_{k}+B\left(\tilde{\theta}, \tilde{\rho}_{k}\right) u_{k}+\tilde{w}_{k}, \\
y_{k} & =C\left(\tilde{\theta}, \tilde{\rho}_{k}\right) x_{k}+D\left(\tilde{\theta}, \tilde{\rho}_{k}\right) u_{k}+\tilde{v}_{k},
\end{aligned}
$$

where $u_{k} \in \mathbb{R}^{n_{u}}, y_{k} \in \mathbb{R}^{n_{y}}, x_{k} \in \mathbb{R}^{n_{x}}, \tilde{w}_{k}$, and $\tilde{v}_{k}$ are the system input, measured output, state, process noise, and measurement noise, respectively. The system parameters $\tilde{\theta} \in \mathbb{R}^{n_{\theta}}$ are unknown. The vector $\tilde{\rho}_{k} \in \mathbb{R}^{n_{\rho}}$ contains the scheduling parameters. The measurement of $\tilde{\rho}_{k}$ is denoted as

$$
\rho_{k}=\tilde{\rho}_{k}+\tilde{\eta}_{k}
$$

which is corrupted by a measurement noise $\tilde{\eta}_{k}$.

Assumption 1. All the system matrices $A\left(\tilde{\theta}, \tilde{\rho}_{k}\right), B\left(\tilde{\theta}, \tilde{\rho}_{k}\right), C\left(\tilde{\theta}, \tilde{\rho}_{k}\right)$ and $D\left(\tilde{\theta}, \tilde{\rho}_{k}\right)$ ${ }_{65}$ in (1) have polynomial dependence on both $\tilde{\theta}$ and $\tilde{\rho}_{k}$.

Assumption 2. The uncertain parameter vector $\tilde{\theta}$ is time invariant (TI), belongs to a bounded set $\Theta$, and is described by TI random variables with known probability density function (PDF) $f_{\theta}$.

Assumption 3. Noises $\tilde{w}_{k}, \tilde{v}_{k}$ in (1) and $\tilde{\eta}_{k}$ in (2) are of zero-mean white 70 nature with known PDFs $f_{w}, f_{v}$, and $f_{\eta}$, respectively; and $\tilde{\theta}, \tilde{w}, \tilde{v}$ and $\tilde{\eta}$ are mutually independent.

Assumption 1 is not restrictive because any nonpolynomial nonlinear dependence on $\tilde{\theta}$ and $\tilde{\rho}_{k}$ can be adequately approximated by polynomials or piecewise polynomials [27. The approximation error can be made arbitrarily small as long 
75 as the approximating polynomial has a sufficiently large degree [28. Therefore, the approximation error is assumed negligible in this paper. Otherwise, another uncertain variable should be introduced to fully account for this approximation error, which often leads to an increased MDR.

In Assumption 2 time invariance of $\tilde{\theta}$ can be justified because physical paso rameters generally have slow variations, and are usually considered as TI over a finite-time horizon [16. The PDF of $\tilde{\theta}$ can be obtained by either offline identification from data $29,30,31$, or a priori knowledge that specifies the relative importance of different points in the uncertainty region $\Theta$. The PDFs of $\tilde{\theta}, \tilde{w}$, $\tilde{v}$ and $\tilde{\eta}$ can be non-Gaussian, which is not restricted by Assumption 3

For the sake of brevity, let $A\left(\tilde{\theta}, \tilde{\rho}_{k}\right), B\left(\tilde{\theta}, \tilde{\rho}_{k}\right), C\left(\tilde{\theta}, \tilde{\rho}_{k}\right)$ and $D\left(\tilde{\theta}, \tilde{\rho}_{k}\right)$ be denoted by $A_{k}, B_{k}, C_{k}$, and $D_{k}$, respectively. From (1) and (2), the stacked system equations over a time window $[k-h+1, k]$ of length $h$ can be expressed as

$$
\begin{aligned}
\mathbf{y}_{k, h} & =\mathcal{O}\left(\tilde{\boldsymbol{\xi}}_{k}\right) x_{k-h+1}+\mathcal{H}_{u}\left(\tilde{\boldsymbol{\xi}}_{k}\right) \mathbf{u}_{k, h}+\mathcal{H}_{w}\left(\tilde{\boldsymbol{\xi}}_{k}\right) \tilde{\mathbf{w}}_{k, h}+\tilde{\mathbf{v}}_{k, h}, \\
\boldsymbol{\rho}_{k, h} & =\tilde{\boldsymbol{\rho}}_{k, h}+\tilde{\boldsymbol{\eta}}_{k, h},
\end{aligned}
$$

where

$$
\begin{gathered}
\mathbf{y}_{k, h}=\left[\begin{array}{c}
y_{k-h+1} \\
y_{k-h+2} \\
\vdots \\
y_{k}
\end{array}\right], \tilde{\boldsymbol{\xi}}_{k}=\left[\begin{array}{c}
\tilde{\theta} \\
\tilde{\boldsymbol{\rho}}_{k, h}
\end{array}\right], \mathcal{O}\left(\tilde{\boldsymbol{\xi}}_{k}\right)=\left[\begin{array}{c}
C_{k-h+1} \\
C_{k-h+2} A_{k-h+1} \\
\vdots \\
C_{k} \prod_{i=1}^{h-1} A_{k-i}
\end{array}\right], \\
\mathcal{H}_{u}\left(\tilde{\boldsymbol{\xi}}_{k}\right)=\left[\begin{array}{cccc}
D_{k-h+1} & 0 & \cdots & 0 \\
C_{k-h+2} B_{k-h+1} & D_{k-h+2} & \ddots & \vdots \\
\vdots & \vdots & \ddots & 0 \\
C_{k} \prod_{i=1}^{h-2} A_{k-i} B_{k-h+1} & C_{k} \prod_{i=1}^{h-3} A_{k-i} B_{k-h+2} & \cdots & D_{k}
\end{array}\right],
\end{gathered}
$$

${ }_{85} \mathbf{u}_{k, h}, \tilde{\boldsymbol{\rho}}_{k, h}, \tilde{\mathbf{w}}_{k, h}, \tilde{\mathbf{v}}_{k, h}$, and $\tilde{\boldsymbol{\eta}}_{k, h}$ are defined similarly to $\mathbf{y}_{k, h}$, and $\mathcal{H}_{w}\left(\tilde{\boldsymbol{\xi}}_{k}\right)$ is defined similarly to $\mathcal{H}_{u}\left(\tilde{\boldsymbol{\xi}}_{k}\right)$.

In an SM FD method, the detection of faults relies on invalidating a fault-free model [14, 23]. Specifically, a fault alarm is reported as long as the measured output $y_{k}$ cannot be generated by the fault-free model (1) and its associated 90 uncertainty descriptions, given the system input $u_{k}$ and the measured scheduling parameter $\rho_{k}$. Such an invalidation approach does not require any fault model, thus is not restricted to any particular fault type [14, 23]. The conventional deterministic SM approach adopts the unknown-but-bounded uncertainty description [14, 23]. In contrast, this paper aims at exploiting the probabilis-

95 tic information of uncertainties to further reduce the MDR by admitting an acceptable FAR. 


\section{Overview of probabilistic SM parity relation approach}

The main idea of the proposed approach is explained in this section.

According to (3), the observability matrix $\mathcal{O}\left(\tilde{\boldsymbol{\xi}}_{k}\right)$ polynomially depends on $\tilde{\boldsymbol{\xi}}_{k}$ which itself includes the unknown parameter $\tilde{\theta}$ and the scheduling parameters $\tilde{\boldsymbol{\rho}}_{k, h}$. Therefore, $\mathcal{O}\left(\tilde{\boldsymbol{\xi}}_{k}\right)$ is unknown and time-varying, which does not allow the use of a conventional time-invariant parity matrix to decouple the unknown initial state $x_{k-h+1}$ in (3a). Alternatively, a polynomial parity matrix $V\left(\tilde{\boldsymbol{\xi}}_{k}\right)$ is first constructed in Section 4 to satisfy

$$
V\left(\tilde{\boldsymbol{\xi}}_{k}\right) \mathcal{O}\left(\tilde{\boldsymbol{\xi}}_{k}\right)=0 \text { for any } \tilde{\boldsymbol{\xi}}_{k},
$$

so that the premultiplication of 3 by $V\left(\tilde{\boldsymbol{\xi}}_{k}\right)$ decouples the unknown initial term $\mathcal{O}\left(\tilde{\boldsymbol{\xi}}_{k}\right) x_{k-h+1}$ and generates the parity relation

$$
V\left(\tilde{\boldsymbol{\xi}}_{k}\right)\left(\mathbf{y}_{k, h}-\mathcal{H}_{u}\left(\tilde{\boldsymbol{\xi}}_{k}\right) \mathbf{u}_{k, h}-\mathcal{H}_{w}\left(\tilde{\boldsymbol{\xi}}_{k}\right) \tilde{\mathbf{w}}_{k, h}-\tilde{\mathbf{v}}_{k, h}\right)=0 .
$$

In the parity relation (6), the true parameter $\tilde{\boldsymbol{\xi}}_{k}$ and noises $\tilde{\mathbf{w}}_{k, h}, \tilde{\mathbf{v}}_{k, h}$, and $\tilde{\boldsymbol{\eta}}_{k, h}$ are unavailable. Let $\theta, \mathbf{w}_{k, h}, \mathbf{v}_{k, h}$, and $\boldsymbol{\eta}_{k, h}$ represent random realizations of $\tilde{\theta}, \tilde{\mathbf{w}}_{k, h}, \tilde{\mathbf{v}}_{k, h}$, and $\tilde{\boldsymbol{\eta}}_{k, h}$, respectively. Then, by defining the parameter vector

$$
\boldsymbol{\xi}_{k}=\left[\begin{array}{ll}
\theta^{\top} & \left(\boldsymbol{\rho}_{k, h}-\boldsymbol{\eta}_{k, h}\right)^{\top}
\end{array}\right]^{\top}
$$

according to (3b), a residual can be expressed as a function of the considered uncertainties, i.e.,

$$
\begin{aligned}
\mathbf{r}_{k}(\boldsymbol{\xi}, \mathbf{w}, \mathbf{v}) & =V\left(\boldsymbol{\xi}_{k}\right)\left(\mathbf{y}_{k, h}-\mathcal{H}_{u}\left(\boldsymbol{\xi}_{k}\right) \mathbf{u}_{k, h}-\mathcal{H}_{w}\left(\boldsymbol{\xi}_{k}\right) \mathbf{w}_{k, h}-\mathbf{v}_{k, h}\right) \\
& =\mathcal{G}\left(\boldsymbol{\xi}_{k}\right) \mathbf{z}_{k, h}-\mathcal{J}\left(\boldsymbol{\xi}_{k}\right) \mathbf{n}_{k, h}
\end{aligned}
$$

with

$$
\begin{aligned}
\mathcal{G}\left(\boldsymbol{\xi}_{k}\right) & =\left[\begin{array}{ll}
V\left(\boldsymbol{\xi}_{k}\right) & -V\left(\boldsymbol{\xi}_{k}\right) \mathcal{H}_{u}\left(\boldsymbol{\xi}_{k}\right)
\end{array}\right], \mathbf{z}_{k, h}=\left[\begin{array}{ll}
\mathbf{y}_{k, h}^{\top} & \mathbf{u}_{k, h}^{\top}
\end{array}\right]^{\top}, \\
\mathcal{J}\left(\boldsymbol{\xi}_{k}\right) & =\left[\begin{array}{ll}
V\left(\boldsymbol{\xi}_{k}\right) & V\left(\boldsymbol{\xi}_{k}\right) \mathcal{H}_{w}\left(\boldsymbol{\xi}_{k}\right)
\end{array}\right], \mathbf{n}_{k, h}=\left[\begin{array}{ll}
\mathbf{v}_{k, h}^{\top} & \mathbf{w}_{k, h}^{\top}
\end{array}\right]^{\top} .
\end{aligned}
$$

Note that the residual $\mathbf{r}_{k}$ is random, since it is computed by using random samples of uncertain variables $\boldsymbol{\xi}, \mathbf{w}, \mathbf{v}$. Accordingly, a confidence set $\Delta_{k}$ with a confidence level $\gamma$ for $\mathbf{r}_{k}$, i.e.,

$$
\operatorname{Pr}\left\{\mathbf{r}_{k}(\boldsymbol{\xi}, \mathbf{w}, \mathbf{v}) \in \Delta_{k} \mid \theta \sim f_{\theta}, w_{k} \sim f_{w}, v_{k} \sim f_{v}, \eta_{k} \sim f_{\eta}\right\} \geq \gamma,
$$

is constructed in Section 5 by exploiting the distributional information of con-

sidered uncertainties, where $\operatorname{Pr}\{\cdot\}$ represents the probability of the associated event.

With the above derivations, the following FD logic is adopted:

$$
\left\{\begin{array}{l}
\mathbf{0} \in \Delta_{k} \Rightarrow \text { the monitored system is fault-free } \\
\mathbf{0} \notin \Delta_{k} \Rightarrow \text { the monitored system is faulty. }
\end{array}\right.
$$

Note that $\mathbf{0}$ in (11) is a zero vector whose dimension is the same as $\mathbf{r}_{k}$. 
Although this FD logic seems similar to the deterministic SM approaches in [15. 16], there exists a significant difference: the deterministic SM approaches rely on an overbounding convex set to ensure zero FAR, while the confidence set $\Delta_{k}$ used in (11) is obtained via stochastic uncertainty propagation and could be non-convex as discussed in Section 5 .

\section{Computing a polynomial parity matrix}

This section presents a method for computing the polynomial parity matrix $V\left(\tilde{\boldsymbol{\xi}}_{\boldsymbol{k}}\right)$, and discusses how the non-uniqueness of $V\left(\tilde{\boldsymbol{\xi}}_{\boldsymbol{k}}\right)$ affects FD performance.

For the ease of understanding, first we consider the case with only a scalar $\tilde{\theta}$ and no scheduling parameter $\tilde{\rho}_{k}$. In this case, the system matrices are $A(\tilde{\theta})$ and $C(\tilde{\theta})$, and the corresponding observability matrix becomes $\mathcal{O}(\tilde{\theta})$. Let the degrees of the polynomial matrices $A(\tilde{\theta})$ and $C(\tilde{\theta})$ be denoted by $d_{A}$ and $d_{C}$, respectively. Then the observability matrix $\mathcal{O}(\tilde{\theta})$ can be expressed as

$$
\mathcal{O}(\tilde{\theta})=\sum_{j=0}^{d_{O}} O_{j} \tilde{\theta}^{j}
$$

with degree

$$
d_{O}=d_{C}+(h-1) d_{A}
$$

When a polynomial parity matrix

$$
V(\tilde{\theta})=\sum_{i=0}^{d_{V}} V_{i} \tilde{\theta}^{i}
$$

is selected with degree $d_{V}, 5$ becomes

$$
V(\tilde{\theta}) \mathcal{O}(\tilde{\theta})=\left(\sum_{i=0}^{d_{V}} V_{i} \tilde{\theta}^{i}\right)\left(\sum_{j=0}^{d_{O}} O_{j} \tilde{\theta}^{j}\right)=0
$$

The multiplication on the left-hand side of 14 is a polynomial with degree $d_{V}+d_{O}$, whose monomial with degree $i$ is $\sum_{j=0}^{r} V_{j} O_{i-j} \tilde{\theta}^{i}$. All of its monomial coefficients need to be zero, i.e., $\sum_{j=0}^{i} V_{j} O_{i-j}=0$, so that 14 holds for any $\tilde{\theta}$. 
Therefore, (14) is equivalent to

$$
\begin{aligned}
& \mathcal{V} \mathcal{M}=0 \text {, with } \\
& \mathcal{V}=\left[\begin{array}{llll}
V_{0} & V_{1} & \cdots & V_{d_{V}}
\end{array}\right] \text {, } \\
& \mathcal{M}=\left[\begin{array}{cccccccccc}
O_{0} & O_{1} & \cdots & O_{d_{O}} & 0 & \cdots & \cdots & 0 & \cdots & 0 \\
0 & O_{0} & \cdots & O_{d_{O_{O}}} & O_{d_{O}} & 0 & \cdots & 0 & \cdots & 0 \\
\vdots & \ddots & \ddots & \vdots & \vdots & \ddots & \ddots & \vdots & \ddots & \vdots \\
0 & \cdots & 0 & O_{0} & O_{1} & \cdots & O_{d_{O}} & 0 & \cdots & 0 \\
0 & \cdots & 0 & 0 & O_{0} & \cdots & O_{d_{O^{-1}}} & O_{d_{O}} & \cdots & 0 \\
\vdots & \ddots & \vdots & \vdots & \ddots & \ddots & \vdots & \vdots & \ddots & \vdots \\
0 & \cdots & 0 & 0 & \cdots & 0 & O_{0} & O_{1} & \cdots & O_{d_{O}}
\end{array}\right]=0 \text { if } d_{V} \geq d_{O} \\
& \mathcal{M}=\left[\begin{array}{ccccccccccc}
O_{0} & O_{1} & \cdots & O_{d_{V}-1} & O_{d_{V}} & \cdots & O_{d_{O}} & 0 & \cdots & \cdots & 0 \\
0 & O_{0} & O_{1} & \cdots & O_{d_{V}-1} & \cdots & O_{d_{O}-1} & O_{d_{O}} & 0 & \cdots & 0 \\
\vdots & \ddots & \ddots & \ddots & \vdots & \ddots & \vdots & \vdots & \ddots & \ddots & \vdots \\
0 & \cdots & 0 & O_{0} & O_{1} & \cdots & O_{d_{O}-d_{V}+1} & O_{d_{O}-d_{V}+2} & \cdots & O_{d_{O}} & 0 \\
0 & \cdots & \cdots & 0 & O_{0} & \cdots & O_{d_{O}-d_{V}} & O_{d_{O}-d_{V}+1} & \cdots & O_{d_{O}-1} & O_{d_{O}}
\end{array}\right]=0 \\
& \text { if } d_{V}<d_{O} \text {. }
\end{aligned}
$$

The multiplication between $\mathcal{V}$ and the $i^{\text {th }}$ block-column of $\mathcal{M}$ corresponds to the coefficient of the monomial basis with degree $i-1$ in $V(\tilde{\theta}) \mathcal{O}(\tilde{\theta})$. Hence, the size of $\mathcal{M}$ is $m_{\mathcal{M}} \times n_{\mathcal{M}}$, where $m_{\mathcal{M}}=\left(d_{V}+1\right) h n_{y}$, and $n_{\mathcal{M}}=\left(d_{V}+d_{O}+1\right) n_{x}$ is determined by the degree of $V(\tilde{\theta}) \mathcal{O}(\tilde{\theta})$. The solution $\mathcal{V}$ to $115 \mathrm{a}$ can be expressed by $(19)$ that will be explained later on. The block-columns $\left\{V_{i}\right\}$ of $\mathcal{V}$ are then used to construct the polynomial parity matrix $V(\tilde{\theta})$ according to $(13)$.

Next, we proceed with the general case that includes both multi-dimensional $\tilde{\theta}$ and scheduling parameters $\tilde{\boldsymbol{\rho}}_{k, h}$. In this case, the dimension of $\tilde{\boldsymbol{\xi}}_{k}$ defined in (4) is $n_{\xi}=n_{\theta}+h n_{\rho}$. Let $\tilde{\xi}_{k, i}$ denote the $i^{\text {th }}$ element of $\tilde{\boldsymbol{\xi}}_{k}$, and $\tilde{\boldsymbol{\xi}}_{k}^{\varpi}$ a monomial $\tilde{\xi}_{k, 1}^{\varpi_{1}} \tilde{\xi}_{k, 2}^{\varpi_{2}} \cdots \tilde{\xi}_{k, n_{\xi}}^{\varpi_{n_{\xi}}}$, where $\varpi_{i}$ is a nonnegative integer, and $\varpi=$ $\left[\begin{array}{ll}\varpi_{1}^{\top} & \varpi_{2}^{\top} \cdots, \varpi_{n_{\xi}}^{\top}\end{array}\right]^{\top}$ is the multivariate monomial exponent. Then, any polynomial matrix $M\left(\tilde{\boldsymbol{\xi}}_{k}\right)$ can be expressed as

$$
M\left(\tilde{\boldsymbol{\xi}}_{k}\right)=\sum_{\varpi \in \pi\left(M\left(\tilde{\boldsymbol{\xi}}_{k}\right)\right)} M_{[\varpi]} \tilde{\boldsymbol{\xi}}_{k}^{\varpi},
$$

where $M_{[\varpi]}$ is the coefficient matrix for the monomial $\tilde{\xi}_{k}^{\varpi}$, and $\pi\left(M\left(\tilde{\boldsymbol{\xi}}_{k}\right)\right)$ represents the set of all monomial exponents included in $M\left(\tilde{\boldsymbol{\xi}}_{k}\right)$. The degree of a monomial $\tilde{\boldsymbol{\xi}}_{k}^{\varpi}$ is defined by $\sum_{i=1}^{n_{\xi}} \varpi_{i}$, while the degree of a polynomial matrix $M\left(\tilde{\boldsymbol{\xi}}_{k}\right)$ is determined by the maximal degree of the included monomials, i.e., $\max _{\varpi \in \pi\left(M\left(\tilde{\boldsymbol{\xi}}_{k}\right)\right)} \sum_{i=1}^{n_{\xi}} \varpi_{i}$. If the degree of $M\left(\tilde{\boldsymbol{\xi}}_{k}\right)$ is $d_{M}$, the maximum number of monomial terms in $M\left(\tilde{\boldsymbol{\xi}}_{k}\right)$ is

$$
g\left(d_{M}, n_{\xi}\right)=\frac{\left(d_{M}+n_{\xi}\right) !}{d_{M} ! n_{\xi} !} .
$$


Based on these definitions, (5) can be expressed as

$$
\left(\sum_{\varpi \in \pi\left(V\left(\tilde{\boldsymbol{\xi}}_{k}\right)\right)} V_{[\varpi]} \tilde{\xi}_{k}^{\varpi}\right)\left(\sum_{\varphi \in \pi\left(\mathcal{O}\left(\tilde{\boldsymbol{\xi}}_{k}\right)\right)} O_{[\varphi]} \tilde{\xi}_{k}^{\varphi}\right)=0 .
$$

Since each monomial coefficient matrix on the left-hand side of (5) is null, (17) is equivalent to

$$
\sum_{\varpi \in \pi\left(V\left(\tilde{\boldsymbol{\xi}}_{k}\right)\right)} V_{[\varpi]} \mathcal{O}_{[\sigma-\varpi]}=0, \sigma \in \pi\left(V\left(\tilde{\boldsymbol{\xi}}_{k}\right) \mathcal{O}\left(\tilde{\boldsymbol{\xi}}_{k}\right)\right) .
$$

From this equation, $\mathcal{V M}=0$ can be constructed similarly to 15 as follows. Each block in $\mathcal{V}$ is $V_{[\varpi]}$. Each block-column of $\mathcal{M}$ includes the coefficients

$$
\left\{\mathcal{O}_{[\sigma-\varpi]}, \varpi \in \pi\left(V\left(\tilde{\boldsymbol{\xi}}_{k}\right)\right)\right\}
$$

of monomials $\left\{\tilde{\boldsymbol{\xi}}_{\boldsymbol{k}}^{\sigma-\varpi}\right\}$ in $\mathcal{O}\left(\tilde{\boldsymbol{\xi}}_{\boldsymbol{k}}\right)$, and its multiplication with $\mathcal{V}$ produces the monomial coefficient of $\tilde{\boldsymbol{\xi}}_{k}^{\sigma}$ in $V\left(\tilde{\boldsymbol{\xi}}_{k}\right) \mathcal{O}\left(\tilde{\boldsymbol{\xi}}_{k}\right)$. The size of $\mathcal{M}$ is $m_{\mathcal{M}} \times n_{\mathcal{M}}$, with $m_{\mathcal{M}}=g\left(d_{V}, n_{\xi}\right) h n_{y}$ and $n_{\mathcal{M}}=g\left(d_{V}+d_{O}, n_{\xi}\right) n_{x}$.

The existence of a solution to $\mathcal{V M}=0$ is equivalent to the existence of a left nullspace of $\mathcal{M}$. It can be guaranteed by a sufficient condition $g\left(d_{V}, n_{\xi}\right) h n_{y}>$ $g\left(d_{V}+d_{O}, n_{\xi}\right) n_{x}$, i.e., the matrix $\mathcal{M}$ has more rows than columns. Using (16), this sufficient condition can be rewritten as

$$
\prod_{i=1}^{n_{\xi}}\left(1-\frac{d_{O}}{d_{V}+d_{O}+i}\right)>\frac{n_{x}}{h n_{y}} .
$$

Since the left-hand side of 180 is smaller than 1 , the inequality $h n_{y}>n_{x}$ is a necessary condition for (18) to hold. For a fixed horizon length $h$, the existence of a polynomial parity matrix $V\left(\tilde{\boldsymbol{\xi}}_{k}\right)$ is guaranteed by selecting a sufficiently high degree $d_{V}$. Increasing either $h$ or $d_{V}$ results in higher computational cost for both offline design and online residual evaluation. Therefore, the shortest 125 horizon length $h$ and the lowest degree $d_{V}$ are sought by iteratively increasing either $h$ or $d_{V}$ by one until a solution to $\mathcal{V} \mathcal{M}=0$ is found.

Let $r_{\mathcal{M}}$ represent the rank of $\mathcal{M}$. Let $\mathcal{M}_{\perp} \in \mathbb{R}^{m_{\mathcal{M}} \times\left(m_{\mathcal{M}}-r_{\mathcal{M}}\right)}$ denote the orthogonal basis of the left nullspace of $\mathcal{M}$, i.e., $\mathcal{M}_{\perp}^{\top} \mathcal{M}=0$ and $\mathcal{M}_{\perp}^{\top} \mathcal{M}_{\perp}=I$, and it can be computed via singular value decomposition [32]. Then, the solution to $\mathcal{V M}=0$ is expressed as

$$
\mathcal{V}=\Omega \mathcal{M}_{\perp}^{\top}
$$

where $\Omega \in \mathbb{R}^{n_{r} \times\left(m_{\mathcal{M}}-r_{\mathcal{M}}\right)}$ has to be determined. Without loss of generality, $\Omega$ is assumed to be full row rank. As will be presented by the following propositions, any nonsingular matrix $\Omega$ results in the same FAR and FDR, and gives a better 
Define $\mathcal{V}^{\star}=\mathcal{M}_{\perp}^{\top}$, then the corresponding polynomial parity matrix and generated residual are denoted as $V^{\star}(\boldsymbol{\xi})$ and $\mathbf{r}_{k}^{\star}$, respectively, where $\mathbf{r}_{k}^{\star}$ is defined as in (8) by replacing $V\left(\boldsymbol{\xi}_{\boldsymbol{k}}\right)$ with $V^{\star}\left(\boldsymbol{\xi}_{k}\right)$. Define a closed confidence set $\Delta_{k}^{\star}$ for $\mathbf{r}_{k}^{\star}$ by following 10 . Assume that this closed set $\Delta_{k}^{\star}$ is described by $\Delta_{k}^{\star}=$ $\left\{\mathbf{r}_{k}^{\star} \mid \mu\left(\mathbf{r}_{k}^{\star}\right) \leq 0\right\}$, where $\mu: \mathbb{R}^{n_{r}} \mapsto \mathbb{R}^{q}$ is a continuous vector-valued function.

Proposition 1. If $\Omega$ is nonsingular, the achieved FAR and MDR do not depend on the selection of $\Omega$.

Proof. With the chosen $\Omega$, we have $\mathcal{V}=\Omega \mathcal{V}^{\star}, V\left(\boldsymbol{\xi}_{k}\right)=\Omega V^{\star}\left(\boldsymbol{\xi}_{k}\right)$, and $\mathbf{r}_{k}=\Omega \mathbf{r}_{k}^{\star}$ according to (8) and (19). Therefore, the confidence sets $\Delta_{k}^{\star}=\left\{\mathbf{r}_{k}^{\star} \mid \mu\left(\mathbf{r}_{k}^{\star}\right) \leq 0\right\}$ and $\Delta_{k}=\left\{\mathbf{r}_{k} \mid \mu\left(\Omega^{-1} \mathbf{r}_{k}\right) \leq 0\right\}$ give the same FAR and MDR, because $\mu\left(\mathbf{r}_{k}^{\star}\right)=$ $\mu\left(\Omega^{-1} \mathbf{r}_{k}\right)$ holds in any case.

Proposition 2. If $\Omega$ is column-rank deficient, the resulting parity relation achieves a lower FAR but a higher MDR, compared to the selection $\mathcal{V}^{\star}=\mathcal{M}_{\perp}^{\top}$.

Proof. According to (8), the residual $\mathbf{r}_{k}^{\star}(\boldsymbol{\xi}, \mathbf{w}, \mathbf{v})$ generated by using $\mathcal{V}^{\star}$ can be rewritten as

$$
\mathbf{r}_{k}^{\star}(\boldsymbol{\xi}, \mathbf{w}, \mathbf{v})=\overline{\mathbf{r}}_{k}^{\star}(\boldsymbol{\xi}, \mathbf{w}, \mathbf{v})+V^{\star}\left(\boldsymbol{\xi}_{k}\right) \mathbf{f}_{k},
$$

where $\overline{\mathbf{r}}_{k}^{\star}$ is the fault-free part, and $\mathbf{f}_{k}$ describes the contribution of latent faults. When using $\mathcal{V}=\Omega \mathcal{V}^{\star}$ to generate a residual $\mathbf{r}_{k}(\boldsymbol{\xi}, \mathbf{w}, \mathbf{v}), \mathbf{r}_{k}=\Omega \mathbf{r}_{k}^{\star}$ holds. Then it follows from (20) that $\mathbf{r}_{k}$ can be similarly decomposed as $\mathbf{r}_{k}=\Omega \overline{\mathbf{r}}_{k}^{\star}+\Omega V^{\star}\left(\boldsymbol{\xi}_{k}\right) \mathbf{f}_{k}$. Since $\Omega$ is column-rank deficient, there might exist $\boldsymbol{\xi}_{k}$ such that the set of undetectable faults $\left\{\mathbf{f}_{k} \mid \Omega V^{\star}\left(\boldsymbol{\xi}_{k}\right) \mathbf{f}_{k}=0\right\}$ is not null, thus increasing the MDR achieved by using $\mathcal{V}=\Omega \mathcal{V}^{\star}$. For the same reason, it is possible in the fault-free case that a residual $\overline{\mathbf{r}}_{k}^{\star}$ giving a false alarm lies in the null space of $\Omega$, which reduces the FAR achieved by $\mathbf{r}_{k}$.

Remark 1. The Cayley-Hamilton theorem was exploited in [33] to construct a polynomial parity vector for linear time-invariant systems with parametric uncertainties, which is not applicable for the uncertain LPV systems considered in this paper.

Remark 2. How the FD performance of the proposed approach depends on the horizon length $h$ and the degree $d_{V}$ is illustrated in Section 6.3 via Monte Carlo simulations. It can be observed that (i) the MDR becomes higher when $d_{V}$ is larger than its minimal value, and (ii) the MDR decreases as $h$ increases. $A$ rigorous proof for these observations is left to future work.

Remark 3. For the iteration from $h$ to $h+1$ or from $d_{V}$ to $d_{V}+1$, there exist methods to incrementally update $\mathcal{M}_{\perp}$, without computing it from scratch. As $h$ or $d_{V}$ increases by one, additional rows and columns are inserted into $\mathcal{M}$, according to (15c), (15d), and (17). This feature allows incrementally updating the $S V D$ of $\mathcal{M}$, see [34 as an example. 


\section{Computing the residual distribution and its confidence set}

Since the FD logic (11) requires the confidence set $\Delta_{k}$ defined in $(10)$, the residual distribution has to be quantified. In this section, the residual (8) is first structured as an LTV transformation of an uncertainty vector. Then, the residual distribution and its confidence set $\Delta_{k}$ defined in 100 are computed using a GM approach.

\subsection{Residual in an LTV structure}

According to Assumption 1, $\mathcal{G}\left(\boldsymbol{\xi}_{\boldsymbol{k}}\right)$ and $\mathcal{J}\left(\boldsymbol{\xi}_{\boldsymbol{k}}\right)$ defined in (9) are polynomial matrices depending on $\theta, \boldsymbol{\eta}_{k, h}$, and $\boldsymbol{\rho}_{k, h}$. Hence $\mathcal{G}\left(\boldsymbol{\xi}_{\boldsymbol{k}}\right)$ and $\mathcal{J}\left(\boldsymbol{\xi}_{\boldsymbol{k}}\right)$ can be expressed as

$$
\begin{aligned}
& \mathcal{G}\left(\boldsymbol{\xi}_{k}\right)=\sum_{i=0}^{N} G_{i}\left(\theta, \boldsymbol{\eta}_{k, h}\right) \psi_{i}\left(\boldsymbol{\rho}_{k, h}\right), \\
& \mathcal{J}\left(\boldsymbol{\xi}_{k}\right)=\sum_{i=0}^{N} J_{i}\left(\theta, \boldsymbol{\eta}_{k, h}\right) \psi_{i}\left(\boldsymbol{\rho}_{k, h}\right),
\end{aligned}
$$

which are regarded as polynomials in terms of $\boldsymbol{\rho}_{k, h}:\left\{\psi_{i}\left(\boldsymbol{\rho}_{k, h}\right)\right\}$ are the monomial bases, and $\left\{G_{i}\left(\theta, \boldsymbol{\eta}_{k, h}\right)\right\}$ and $\left\{J_{i}\left(\theta, \boldsymbol{\eta}_{k, h}\right)\right\}$ are the corresponding coefficients. The number $N$ of the monomial bases $\left\{\psi_{i}\left(\boldsymbol{\rho}_{k, h}\right)\right\}$ is determined by the dimension of $\boldsymbol{\rho}_{k, h}$ as well as the highest degree of $\boldsymbol{\rho}_{k, h}$ in $\left\{G_{i}\left(\theta, \boldsymbol{\eta}_{k, h}\right)\right\}$ and $\left\{J_{i}\left(\theta, \boldsymbol{\eta}_{k, h}\right)\right\}$. As long as the polynomial matrices $\mathcal{G}\left(\boldsymbol{\xi}_{\boldsymbol{k}}\right)$ and $\mathcal{J}\left(\boldsymbol{\xi}_{\boldsymbol{k}}\right)$ are obtained, $\left\{G_{i}\left(\theta, \boldsymbol{\eta}_{k, h}\right)\right\}$ and $\left\{J_{i}\left(\theta, \boldsymbol{\eta}_{k, h}\right)\right\}$ can be determined according to their polynomial structure. For instance,

$$
\left[\begin{array}{l}
\theta+\eta \rho^{2} \\
2+\eta^{2} \rho
\end{array}\right]=\left[\begin{array}{l}
\theta \\
2
\end{array}\right]+\left[\begin{array}{c}
0 \\
\eta^{2}
\end{array}\right] \rho+\left[\begin{array}{l}
\eta \\
0
\end{array}\right] \rho^{2} .
$$

Let $n_{r}$ be the dimension of the residual vector, $\otimes$ represent the Kronecker product, and $\operatorname{vec}(\cdot)$ denote the vectorization of a matrix by stacking its columns into a single column vector. Then, using (21), the residual in (8) can be rewritten into an LTV form

$$
\begin{aligned}
\mathbf{r}_{k}(\boldsymbol{\xi}, \mathbf{w}, \mathbf{v}) & =\sum_{i=0}^{N}\left\{G_{i}\left(\theta, \boldsymbol{\eta}_{k, h}\right) \mathbf{z}_{k, h} \psi_{i}\left(\boldsymbol{\rho}_{k, h}\right)-J_{i}\left(\theta, \boldsymbol{\eta}_{k, h}\right) \mathbf{n}_{k, h} \psi_{i}\left(\boldsymbol{\rho}_{k, h}\right)\right\} \\
& =\sum_{i=0}^{N}\left\{L_{i, k} \operatorname{vec}\left(G_{i}\left(\theta, \boldsymbol{\eta}_{k, h}\right)\right)-S_{i, k} J_{i}\left(\theta, \boldsymbol{\eta}_{k, h}\right) \mathbf{n}_{k, h}\right\} \\
& =\underbrace{\left[\begin{array}{ll}
\mathcal{L}_{k} & -\mathcal{S}_{k}
\end{array}\right]}_{\boldsymbol{\Gamma}_{k}} \underbrace{\left[\begin{array}{c}
\boldsymbol{\lambda}_{L}\left(\theta, \boldsymbol{\eta}_{k, h}\right) \\
\boldsymbol{\lambda}_{S}\left(\theta, \boldsymbol{\eta}_{k, h}, \mathbf{n}_{k, h}\right)
\end{array}\right]}_{\boldsymbol{\lambda}\left(\theta, \boldsymbol{\eta}_{k, h}, \mathbf{n}_{k, h}\right)},
\end{aligned}
$$


with

$$
\begin{aligned}
& L_{i, k}=\left(\mathbf{z}_{k, h}^{\top} \psi_{i}\left(\boldsymbol{\rho}_{k, h}\right)\right) \otimes I_{n_{r}}, S_{i, k}=\psi_{i}\left(\boldsymbol{\rho}_{k, h}\right) \otimes I_{n_{r}}, \\
& \mathcal{L}_{k}=\left[\begin{array}{llll}
L_{0, k} & L_{1, k} & \cdots & L_{N, k}
\end{array}\right], \mathcal{S}_{k}=\left[\begin{array}{llll}
S_{0, k} & S_{1, k} & \cdots & S_{N, k}
\end{array}\right], \\
& \boldsymbol{\lambda}_{L}\left(\theta, \boldsymbol{\eta}_{k, h}\right)=\left[\begin{array}{c}
\operatorname{vec}\left(G_{0}\left(\theta, \boldsymbol{\eta}_{k, h}\right)\right) \\
\operatorname{vec}\left(G_{1}\left(\theta, \boldsymbol{\eta}_{k, h}\right)\right) \\
\vdots \\
\operatorname{vec}\left(G_{N}\left(\theta, \boldsymbol{\eta}_{k, h}\right)\right)
\end{array}\right], \boldsymbol{\lambda}_{S}\left(\theta, \boldsymbol{\eta}_{k, h}, \mathbf{n}_{k, h}\right)=\left[\begin{array}{c}
J_{0}\left(\theta, \boldsymbol{\eta}_{k, h}\right) \mathbf{n}_{k, h} \\
J_{1}\left(\theta, \boldsymbol{\eta}_{k, h}\right) \mathbf{n}_{k, h} \\
\vdots \\
J_{N}\left(\theta, \boldsymbol{\eta}_{k, h}\right) \mathbf{n}_{k, h}
\end{array}\right] \text {. }
\end{aligned}
$$

The second equation in 22 is derived from the property that

$$
\operatorname{vec}\left(X_{1} X_{2} X_{3}\right)=\left(X_{3}^{\top} \otimes X_{1}\right) \operatorname{vec}\left(X_{2}\right)
$$

holds for matrices $X_{1}, X_{2}$, and $X_{3}$.

With the above derivations, the residual in $(22)$ is expressed as a multiplication between the coefficient matrix $\boldsymbol{\Gamma}_{k}$ and the uncertainty vector $\boldsymbol{\lambda}$. The coefficient matrix $\boldsymbol{\Gamma}_{k}$ in 22 is time-varying, and can be computed with the online data $\mathbf{z}_{k, h}$ and $\boldsymbol{\rho}_{k, h}$ according to (23) and (24). The uncertainty vector $\boldsymbol{\lambda}$ is random, and Section 5.2 will present how to compute its PDF by exploiting the PDFs of the unknown parameter $\theta$, the measurement noise $\boldsymbol{\eta}_{k, h}$ of scheduling parameters, and $\mathbf{n}_{k, h}$ representing the process noise and output measurement noise.

\subsection{Residual distribution and its confidence set}

To construct the FD logic (11), this subsection presents the computation of the residual distribution and its confidence set. Due to its polynomial dependence on uncertain variables, the uncertainty vector $\boldsymbol{\lambda}$ is non-Gaussian distributed. Hence the residual generated by (22) is also non-Gaussian. To enable efficient online computation of the residual distribution, a GM approach is proposed by exploiting GM approximations to the distributions of the uncertainty vector $\boldsymbol{\lambda}$ and the residual $\mathbf{r}_{k}$. The details are as follows.

Since $\boldsymbol{\lambda}\left(\theta, \boldsymbol{\eta}_{k, h}, \mathbf{n}_{k, h}\right)$ in 22 has TI polynomial dependence on stochastic uncertainties $\theta, \boldsymbol{\eta}_{k, h}$, and $\mathbf{n}_{k, h}$, it is a stochastic vector whose PDF is also TI and can be approximated offline by a mixture of Gaussians, i.e.,

$$
p(\boldsymbol{\lambda})=\sum_{j=1}^{K} \pi_{j} \mathcal{N}\left(\boldsymbol{\lambda} ; \mu_{\lambda}^{(j)}, \Sigma_{\lambda}^{(j)}\right),
$$

where $K$ is the number of Gaussian components, the mixing coefficients $\left\{\pi_{j}\right\}$ satisfy $0 \leq \pi_{j} \leq 1$ and $\sum_{j=1}^{K} \pi_{j}=1$, and $\mu_{\lambda}^{(j)}$ and $\Sigma_{\lambda}^{(j)}$ represent the mean and covariance matrix of each Gaussian component, respectively. The offline procedure for constructing the GM distribution 26) is: (i) generate a sufficient 
number of samples $\left\{\theta^{(i)}, \boldsymbol{\eta}_{k, h}^{(i)}, \mathbf{n}_{k, h}^{(i)}\right\}$, and compute $\boldsymbol{\lambda}^{(i)}=\boldsymbol{\lambda}\left(\theta^{(i)}, \boldsymbol{\eta}_{k, h}^{(i)}, \mathbf{n}_{k, h}^{(i)}\right)$ according to 22 and 25); and (ii) determine the GM parameters $\left\{\pi_{j}, \mu_{\lambda}^{(j)}, \Sigma_{\lambda}^{(j)}\right\}$ in (26) by solving the maximum likelihood estimation problem

$$
\max _{\left\{\pi_{j}, \mu_{\lambda}^{(j)}, \Sigma_{\lambda}^{(j)}\right\}} \sum_{i=1}^{N_{\lambda}} \ln \left\{\sum_{j=1}^{K} \pi_{j} \mathcal{N}\left(\boldsymbol{\lambda}^{(i)} ; \mu_{\lambda}^{(j)}, \Sigma_{\lambda}^{(j)}\right)\right\}
$$
the number of samples. The $K$ can be determined by comparing multiple models with different $K$ 's using the Akaike information criterion 35 .

As in the EM algorithm, the subsequent analysis relies on formulating GMs in 260 in terms of a discrete latent variable $\boldsymbol{\omega}$ [35. Let $\boldsymbol{\omega}$ denote a $K$ dimensional binary random vector with each element $\omega_{j}$ satisfying $\omega_{j} \in\{0,1\}$ and $\sum_{j=1}^{K} \omega_{j}=1$, i.e., a particular element $\omega_{j}$ is equal to 1 and all the other elements are null. In order to express the GM distribution $p(\boldsymbol{\lambda})$ as a marginal distribution obtained from the joint distribution $p(\boldsymbol{\lambda}, \boldsymbol{\omega})$, define the marginal distribution $p(\boldsymbol{\omega})$ and the conditional distribution $p(\boldsymbol{\lambda} \mid \boldsymbol{\omega})$ as $p\left(\omega_{j}=1\right)=\pi_{j}$ and $p\left(\boldsymbol{\lambda} \mid \omega_{j}=1\right)=\mathcal{N}\left(\boldsymbol{\lambda} ; \mu_{\lambda}^{(j)}, \Sigma_{\lambda}^{(j)}\right)$, respectively. Therefore, the joint distribution is given by $p(\boldsymbol{\lambda}, \boldsymbol{\omega})=p(\boldsymbol{\omega}) p(\boldsymbol{\lambda} \mid \boldsymbol{\omega})$, and the GM distribution $p(\boldsymbol{\lambda})$ in (26) is then equivalently expressed as

$$
p(\boldsymbol{\lambda})=\sum_{\boldsymbol{\omega}} p(\boldsymbol{\omega}) p(\boldsymbol{\lambda} \mid \boldsymbol{\omega})=\sum_{j=1}^{K} p\left(\omega_{j}=1\right) p\left(\boldsymbol{\lambda} \mid \omega_{j}=1\right) .
$$

This expression explicitly associates every realization of $\boldsymbol{\lambda}$ with a discrete value of $\boldsymbol{\omega}$, i.e., a realization of $\boldsymbol{\lambda}$ is generated from a conditional Gaussian distribution $p\left(\boldsymbol{\lambda} \mid \omega_{j}=1\right)$. This point of view is useful for constructing the residual distribution online.

For the EM algorithm to produce a reliable GM approximation, the number of data samples should be sufficiently large compared to the number of free GM parameters, i.e.,

$$
(K-1)+K n_{\lambda}+\frac{1}{2} K n_{\lambda}\left(n_{\lambda}-1\right),
$$

where $n_{\lambda}$ denotes the dimension of $\boldsymbol{\lambda}, K-1, K n_{\lambda}$, and $\frac{1}{2} K n_{\lambda}\left(n_{\lambda}-1\right)$ are respectively the number of free parameters in the mixing coefficients $\left\{\pi^{(j)}\right\}$, the means $\left\{\mu_{\lambda}^{(j)}\right\}$, and the covariance matrices $\left\{\Sigma_{\lambda}^{(j)}\right\}$. This requirement can be 200 demanding from the computation perspective if $n_{\lambda}$ is large. Actually, $\boldsymbol{\lambda}$ would have a rather high dimension as the window length $h$ grows or the degree $d_{V}$ of the polynomial parity matrix increases, according to the definition of $\boldsymbol{\lambda}$ in 22 . and (25). In addition, in such a high dimensional case, the EM algorithm is prone to numerical ill-conditioning [36]. On one hand, the estimated covariance 205 matrix $\Sigma_{\lambda}^{(j)}$ is more likely to be singular, since the number of samples involved in computing $\Sigma_{\lambda}^{(j)}$ is small compared to the dimension of $\Sigma_{\lambda}^{(j)}[36$. On the other hand, since the elements of $\boldsymbol{\lambda}$ have polynomial dependence of rather different 
degrees on uncertain variables, their numerical values could differ by orders of magnitude, which might also results in an ill-conditioned covariance estimate.

Two remedies are adopted in this paper to address the above issue. Firstly, zero elements may be present in $\boldsymbol{\lambda}$ due to the zero elements of $G_{i}\left(\theta, \boldsymbol{\eta}_{k, h}\right)$ and $J_{i}\left(\theta, \boldsymbol{\eta}_{k, h}\right)$, thus they are removed without any information loss. Secondly, the GM modeling step is performed on the intrinsic low-dimensional subspace of $\boldsymbol{\lambda}$ by first conducting dimensional reduction as a preprocessing step. As a popular 215 method for dimension reduction, principal component analysis (PCA) is used here. Let $\boldsymbol{\Lambda}$ denote the data matrix consisting of the sampled uncertainty vector $\boldsymbol{\lambda}^{(i)}$, i.e., $\boldsymbol{\Lambda}=\left[\begin{array}{llll}\boldsymbol{\lambda}^{(1)} & \boldsymbol{\lambda}^{(2)} & \cdots & \boldsymbol{\lambda}^{\left(N_{\lambda}\right)}\end{array}\right]$. Its singular value decomposition is $\Lambda=\mathcal{U S} \mathcal{V}^{\top}$. By selecting the largest $n_{c}$ singular values that capture a significant portion (e.g., $95 \%$ ) of total variance of $\boldsymbol{\Lambda}$, the approximated data matrix is $\hat{\boldsymbol{\Lambda}}=$ ${ }_{220} \mathcal{U}_{1} \mathcal{S}_{1} \mathcal{V}_{1}^{\top}$, where $\mathcal{U}_{1}$ and $\mathcal{V}_{1}$ consist of the corresponding singular vectors within $\mathcal{U}$ and $\mathcal{V}$, respectively, and $\mathcal{S}_{1}$ represents a diagonal matrix with its diagonal entries being the largest $n_{c}$ singular values. After dimensional reduction, the GM modeling step is carried out for $\mathcal{V}_{1}^{\top}$, which is in a reduced subspace of dimension $n_{c}$, and its estimated GM parameters are denoted by $\left\{\hat{\pi}_{j}, \hat{\mu}^{(j)}, \hat{\Sigma}^{(j)}\right\}$.

225 Accordingly, the GM parameters $\left\{\pi_{j}, \mu_{\lambda}^{(j)}, \Sigma_{\lambda}^{(j)}\right\}$ for $\boldsymbol{\lambda}$ are obtained as $\pi_{j}=\hat{\pi}_{j}$, $\mu_{\lambda}^{(j)}=\mathcal{U}_{1} \mathcal{S}_{1} \hat{\mu}^{(j)}, \Sigma_{\lambda}^{(j)}=\mathcal{U}_{1} \mathcal{S}_{1} \hat{\Sigma}^{(j)} \mathcal{S}_{1}^{\top} \mathcal{U}_{1}^{\top}$.

Remark 4. With the introduced dimensional reduction, the GM model is constructed for $\hat{\boldsymbol{\Lambda}}$ instead of the original sampled data matrix $\boldsymbol{\Lambda}$. Since a small portion of total variance in $\boldsymbol{\Lambda}$ is lost in the dimension reduction, $\hat{\boldsymbol{\Lambda}}$ in the re-

${ }_{230}$ duced space, hence its corresponding GM model, cannot capture some uncertain variations under the fault-free condition. This implies that some fault-free variations are not represented by the GM approximation of the residual distribution, which could lead to false alarms in FD, as will be illustrated by the simulation study in Section 6.3.

235 Remark 5. Other approaches reviewed in [36] can be also utilized for the highdimensional GM modeling, but they are not the focus of this paper.

Remark 6. To validate the GM approximation, the well-known KolmogorovSmirnov distance

$$
\sup _{\boldsymbol{\lambda} \in \mathbb{R}^{n_{\lambda}}}\left|F_{n}(\boldsymbol{\lambda})-\hat{F}(\boldsymbol{\lambda})\right|
$$

can be used to evaluate the goodness-of-fit between the empirical cumulative distribution function $(C D F) F_{n}(\boldsymbol{\lambda})$ of the samples $\left\{\boldsymbol{\lambda}^{(i)}\right\}$ and the CDF of the GM approximation $\hat{F}(\boldsymbol{\lambda})$ [37], with $n_{\boldsymbol{\lambda}}$ denoting the dimension of $\boldsymbol{\lambda}$. Computing 240 the above distance for multivariate distributions is non-trivial, and interested readers are referred to [37] for more details.

From the online measurements $\left\{\mathbf{u}_{k, h}, \mathbf{y}_{k, h}, \boldsymbol{\rho}_{k, h}\right\}, L_{i, k}, S_{i, k}, \mathcal{L}_{k}, \mathcal{S}_{k}$, and $\boldsymbol{\Gamma}_{k}$ can be computed according to their definitions in 22)-24. With $\omega_{j}=1$, the 
conditional distribution of the residual $\mathbf{r}_{k}$ is then derived as

$$
\begin{aligned}
& p\left(\mathbf{r}_{k} \mid \omega_{j}=1\right)=\mathcal{N}\left(\mathbf{r}_{k} ; \mu_{r, k}^{(j)}, \Sigma_{r, k}^{(j)}\right), \\
& \mu_{r, k}^{(j)}=\boldsymbol{\Gamma}_{k} \mu_{\lambda}^{(j)}, \Sigma_{r, k}^{(j)}=\boldsymbol{\Gamma}_{k} \Sigma_{\lambda}^{(j)} \boldsymbol{\Gamma}_{k}^{\top},
\end{aligned}
$$

by performing a linear transformation $(22)$ on the conditional Gaussian component $p\left(\boldsymbol{\lambda} \mid \omega_{j}=1\right)$. Hence, the distribution of the residual can be also approximated by a Gaussian mixture

$$
p\left(\mathbf{r}_{k}\right)=\sum_{j=1}^{K} \pi_{j} \mathcal{N}\left(\mathbf{r}_{k} ; \mu_{r, k}^{(j)}, \Sigma_{r, k}^{(j)}\right) .
$$

Next, the confidence set $\Delta_{k}$ for the random residual $\mathbf{r}_{k}(\boldsymbol{\xi}, \mathbf{w}, \mathbf{v})$ is determined such that 10 holds.

Theorem 3. Given the GM distribution (28) of the random residual $\mathbf{r}_{k}, \operatorname{Pr}\left\{\mathbf{r}_{k} \in\right.$ $\left.\Delta_{k}\right\} \geq \gamma$ holds if the confidence set $\Delta_{k}$ is constructed as

$$
\Delta_{k}=\bigcup_{j=1}^{K} \Delta_{k}^{(j)},
$$

where

$$
\Delta_{k}^{(j)}=\left\{\mathbf{r}_{k} \mid\left(\mathbf{r}_{k}-\mu_{r, k}^{(j)}\right)^{\top}\left(\Sigma_{r, k}^{(j)}\right)^{-1}\left(\mathbf{r}_{k}-\mu_{r, k}^{(j)}\right) \leq \chi_{n_{r}}^{2}(\gamma)\right\},
$$

is an ellipsoidal confidence set defined for the $j^{\text {th }}$ Gaussian component $p\left(\mathbf{r}_{k} \mid \omega_{j}=\right.$ 245 $1)$, and $\chi_{n_{r}}^{2}(\gamma) \in \mathbb{R}$ represents the value whose cumulative probability under the $\chi^{2}$ distribution with $n_{r}$ degrees of freedom is specified by $\gamma$.

Proof. With $\omega_{j}=1, \mathbf{r}_{k}$ follows the conditional Gaussian distribution $p\left(\mathbf{r}_{k} \mid \omega_{j}=\right.$ 1), and $\operatorname{Pr}\left\{\mathbf{r}_{k} \in \Delta_{k} \mid \omega_{j}=1\right\} \geq \operatorname{Pr}\left\{\mathbf{r}_{k} \in \Delta_{k}^{(j)} \mid \omega_{j}=1\right\} \geq \gamma$ holds for $\Delta_{k}$ and $\Delta_{k}^{(j)}$ defined in 29) and (30). Then it follows that

$$
\begin{aligned}
\operatorname{Pr}\left\{\mathbf{r}_{k} \in \Delta_{k}\right\} & =\sum_{j=1}^{K} \operatorname{Pr}\left\{\mathbf{r}_{k} \in \Delta_{k} \mid \omega_{j}=1\right\} \operatorname{Pr}\left\{\omega_{j}=1\right\} \\
& \geq \sum_{j=1}^{K} \operatorname{Pr}\left\{\mathbf{r}_{k} \in \Delta_{k}^{(j)} \mid \omega_{j}=1\right\} \operatorname{Pr}\left\{\omega_{j}=1\right\} \\
& \geq \gamma \sum_{j=1}^{K} \operatorname{Pr}\left\{\omega_{j}=1\right\}=\gamma .
\end{aligned}
$$

Remark 7. Due to the possible overlaps among the confidence sets $\left\{\Delta_{k}^{(j)}\right\}$, the actually achieved probability $\operatorname{Pr}\left\{\mathbf{r}_{k} \in \Delta_{k}\right\}$ can be larger than the predefined confidence level $\gamma$. 
With the confidence set $\Delta_{k}$ defined in (29) and (30), the FD logic (11) amounts to checking whether there exists at least one confidence ellipsoid $\Delta_{k}^{(j)}$ such that $\mathbf{0} \in \Delta_{k}^{(j)}$, or equivalently, $\left(\mu_{r, k}^{(j)}\right)^{\top}\left(\Sigma_{r, k}^{(j)}\right)^{-1}\left(\mu_{r, k}^{(j)}\right) \leq \chi_{n_{r}}^{2}(\gamma)$, holds. This FD logic can be further compactly expressed as

$$
\left\{\begin{array}{l}
\nu_{k} \leq 1 \Rightarrow \text { the monitored system is fault-free } \\
\nu_{k}>1 \Rightarrow \text { the monitored system is faulty }
\end{array}\right.
$$

with

$$
\nu_{k}=\min _{1 \leq j \leq K} \frac{\left(\mu_{r, k}^{(j)}\right)^{\top}\left(\Sigma_{r, k}^{(j)}\right)^{-1}\left(\mu_{r, k}^{(j)}\right)}{\chi_{n_{r}}^{2}(\gamma)} .
$$

The proposed FD approach is summarized in Algorithm 1.

\begin{tabular}{l} 
Algorithm 1 Probability-guaranteed parity relation \\
\hline Offline procedures:
\end{tabular}

1. Select a horizon length $h$ and a degree $d_{V}$. Compute the polynomial parity matrix $V\left(\boldsymbol{\xi}_{k}\right)$ by following Section 4

2. Determine the monomial bases $\left\{\psi_{i}\left(\boldsymbol{\rho}_{k, h}\right), i=0, \cdots, N\right\}$ and the polynomial matrices $\left\{G_{i}\left(\theta, \boldsymbol{\eta}_{k, h}\right), J_{i}\left(\theta, \boldsymbol{\eta}_{k, h}\right)\right\}$ in 21). Note that specific values of $\theta, \boldsymbol{\rho}_{k, h}$, and $\boldsymbol{\eta}_{k, h}$ are not required in this step.

3. Generate a sufficient number of samples $\left\{\theta^{(i)}, \boldsymbol{\eta}_{k, h}^{(i)}, \mathbf{n}_{k, h}^{(i)}\right\}$, compute $\boldsymbol{\lambda}^{(i)}=$ $\boldsymbol{\lambda}\left(\theta^{(i)}, \boldsymbol{\eta}_{k, h}^{(i)}, \mathbf{n}_{k, h}^{(i)}\right)$ according to 22 and 25); determine the GM approximation (26) to the sample distribution of $\left\{\boldsymbol{\lambda}^{(i)}\right\}$ using the EM algorithm.

4. Select the confidence level $\gamma$ in 10 .

Online procedures:

Initialization with $k=h$.

5. Construct $\mathbf{u}_{k, h}, \mathbf{y}_{k, h}, \boldsymbol{\rho}_{k, h}$ over the horizon $[k-h+1, k]$.

6. Compute $L_{i, k}$ and $S_{i, k}$ in (23), $\mathcal{L}_{k}$ and $\mathcal{S}_{k}$ in 24, $\boldsymbol{\Gamma}_{k}$ in 22).

7. Compute $\left\{\mu_{r, k}^{(j)}, \Sigma_{r, k}^{(j)}\right\}$ in 27) for each Gaussian component of the residual distribution.

8. Compute $\nu_{k}$ in (32), and determine the fault presence according to (31).

9. $k \leftarrow k+1$, go to Step 5 .

\section{Case study}

\subsection{Simulation setting and model description}

In the case study, a well-mixed non-isothermal continuous stirred tank reactor is considered. It includes three parallel irreversible elementary exothermic reactions $\mathrm{A} \rightarrow \mathrm{B}, \mathrm{A} \rightarrow \mathrm{U}$, and $\mathrm{A} \rightarrow \mathrm{R}$, where $\mathrm{A}$ is the reactant species, $\mathrm{B}$ is the desired product, and $\mathrm{U}$ and $\mathrm{R}$ are undesired byproducts. The reactant $\mathrm{A}$ is fed 


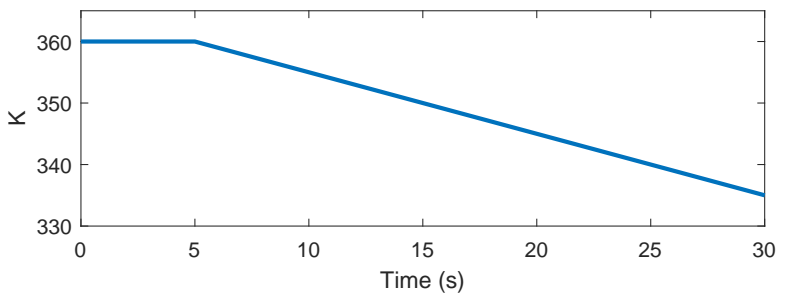

Figure 1: Profile of coolant temperature $T_{\mathrm{cf}}$.

to the reactor at a flow rate $F$, concentration $C_{\mathrm{Af}}$, and temperature $T_{\mathrm{Af}}$. To remove heat from the reactor, a cooling stream at a flow rate $F_{c}$ and temperature $T_{\mathrm{cf}}$ goes into the equipped cooling jacket. The process dynamics takes the form 38]:

$$
\begin{aligned}
\dot{C}_{\mathrm{A}}(t)= & \frac{F}{V}\left(C_{\mathrm{Af}}-C_{\mathrm{A}}(t)\right)-\sum_{i=1}^{3} \kappa_{i}(t) C_{\mathrm{A}}(t), \\
\dot{C}_{\mathrm{B}}(t)= & -\frac{F}{V} C_{\mathrm{B}}(t)+\kappa_{1}(t) C_{\mathrm{A}}(t), \\
\dot{T}(t)= & \frac{F}{V}\left(T_{\mathrm{Af}}-T(t)\right)+\sum_{i=1}^{3} \frac{\left(-\Delta H_{i}+\delta_{H_{i}}\right)}{\rho c_{p}} \kappa_{i}(t) C_{\mathrm{A}}(t) \\
& -\frac{\mathrm{UA}+\delta_{\mathrm{UA}}}{\rho c_{p} V}\left(T(t)-T_{\mathrm{c}}(t)\right), \\
\dot{T}_{\mathrm{c}}(t)= & \frac{F_{c}}{V_{c}}\left(T_{\mathrm{cf}}(t)-T_{\mathrm{c}}(t)\right)+\frac{\mathrm{UA}+\delta_{\mathrm{UA}}}{\rho_{c} c_{p c} V_{c}}\left(T(t)-T_{\mathrm{c}}(t)\right),
\end{aligned}
$$

where $\kappa_{i}(t)$ is defined as

$$
\kappa_{i}(t)=k_{i} \exp \left(\frac{-E_{i}}{R T(t)}\right),
$$

$C_{\mathrm{A}}$ and $C_{\mathrm{B}}$ denote concentrations of the species $\mathrm{A}$ and $\mathrm{B}$ in the reactor, $T$ and $T_{\mathrm{c}}$ denotes temperatures of the reactor and the cooling jacket. The feed concentration and temperature are fixed at $C_{\mathrm{Af}}=4 \mathrm{~mol} / \mathrm{L}$ and $T_{\mathrm{Af}}=360 \mathrm{~K}$, respectively. The initial condition is $C_{\mathrm{A}}(0)=3.5 \mathrm{~mol} / \mathrm{L}, C_{\mathrm{B}}(0)=0 \mathrm{~mol} / \mathrm{L}$, $T(0)=T_{c}(0)=360 \mathrm{~K}$, which is unknown regarding the FD problem. The cooling stream temperature $T_{\mathrm{cf}}$ varies as depicted in Figure 1. With a sampling interval $t_{s}=0.1 \mathrm{~s}$, the sensor outputs $C_{\mathrm{B}}^{m}, T^{m}$, and $T_{\mathrm{c}}^{m}$ are the measurements of the reactor concentration $C_{\mathrm{B}}$, the reactor temperatures $T$ and the cooling jacket temperature $T_{\mathrm{c}}$, with zero-mean Gaussian white noises $v_{C_{\mathrm{B}}}, v_{T}$, and $v_{T_{\mathrm{c}}}$, whose standard deviations are $0.01 \mathrm{~mol} / \mathrm{L}, 0.1 \mathrm{~K}$, and $0.1 \mathrm{~K}$, respectively.

The system parameters are described in Table 1 . As shown in (33), the enthalpies $\left\{\Delta H_{i}\right\}$ and the heat transfer coefficient UA are subject to probabilistic time-invariant uncertainties represented by $\left\{\delta_{H_{i}}\right\}$ and $\delta_{\mathrm{UA}}$, respectively. In this simulation, $\left\{\delta_{H_{i}}\right\}$ are uniformly distributed over the interval $[-100,100] \mathrm{J} / \mathrm{mol}$, 

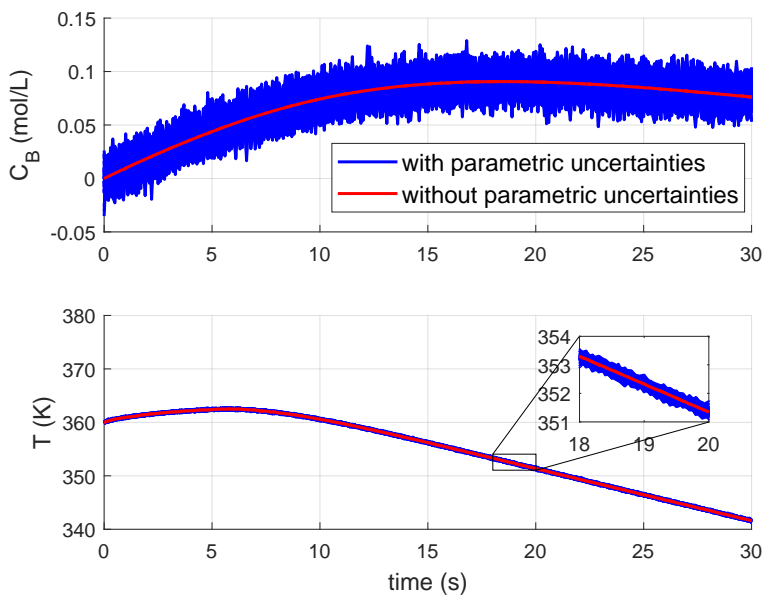

Figure 2: System outputs from 100 fault-free Monte Carlo simulations with and without parametric uncertainties.

while $\delta_{\mathrm{UA}}$ follows a truncated Gaussian distribution which is truncated over $\left[-1 \times 10^{5}, 1 \times 10^{5}\right] \mathrm{J} /(\mathrm{min} \cdot \mathrm{K})$ from a conventional Gaussian distribution with zero mean and standard deviation $1 \times 10^{5} \mathrm{~J} /(\mathrm{min} \cdot \mathrm{K})$. Figure 2 depicts the sensor outputs $C_{B}^{m}(t)$ and $T^{m}(t)$ from 100 fault-free Monte Carlo simulations with and without the above uncertain parameters. The clear difference between faultfree output behaviors in the above two cases implies that neglecting parametric uncertainties would result in severe false alarms.

The following two fault scenarios are considered:

- Sensor bias: the reactor temperature sensor output $T^{m}(t)$ becomes biased by $1.7 \mathrm{~K}$, i.e., $T^{m}(t)=T(t)+1.7+v_{T}(t)$ for $t \geq 15 \mathrm{~s}$.

- Valve stuck: the flow valve of the cooling stream gets stuck at $T_{\mathrm{cf}}=$ $22 \mathrm{~L} / \mathrm{min}$ for $t \geq 15 \mathrm{~s}$.

To account for the nonlinear dependence of $\left\{\kappa_{i}(t)\right\}$ in (34) on $T,\left\{\kappa_{i}(t)\right\}$ over the operation range $T \in[345,365] \mathrm{K}$ are approximated by polynomials $g_{1}\left(T_{k}\right)$, $g_{2}\left(T_{k}\right)$, and $g_{3}\left(T_{k}\right)$ with degrees 5,4 , and 4 , whose maximum absolute approximation errors are $1.05 \times 10^{-10}, 3.06 \times 10^{-12}$, and $3.06 \times 10^{-12}$, respectively. By applying the Euler method and replacing $\left\{\kappa_{i}\right\}$ in (34) with polynomials $\left\{g_{i}(T)\right\}$, the continuous-time system (33) with parameters in Table 1 is transformed into the following discrete-time LPV form whose scheduling parameter is the reactor temperature $T_{k}$, i.e.,

$$
\begin{aligned}
x_{k+1} & =A\left(T_{k}\right) x_{k}+B u_{k}+B_{w} w_{0 k}, \\
y_{k} & =C x_{k}+D_{v} v_{0 k}
\end{aligned}
$$


Table 1: System parameters

\begin{tabular}{|c|c|c|c|}
\hline Parameter & Description & Value & Unit \\
\hline$F$ & Inlet flow rate & 83.3 & $\mathrm{~L} / \mathrm{min}$ \\
\hline$F_{c}$ & Flow rate of cooling stream & 50 & $\mathrm{~L} / \min$ \\
\hline$V$ & Tank volume & 1000 & $\mathrm{~L}$ \\
\hline$V_{c}$ & Volume of cooling jacket & 100 & $\mathrm{~L}$ \\
\hline$\Delta H_{1}$ & Enthalpy of $\mathrm{A} \rightarrow \mathrm{B}$ & $-5 \times 10^{4}$ & $\mathrm{~J} / \mathrm{mol}$ \\
\hline$\Delta H_{2}$ & Enthalpy of $\mathrm{A} \rightarrow \mathrm{U}$ & $-5.2 \times 10^{4}$ & $\mathrm{~J} / \mathrm{mol}$ \\
\hline$\Delta H_{3}$ & Enthalpy of $\mathrm{A} \rightarrow \mathrm{R}$ & $-5.4 \times 10^{4}$ & $\mathrm{~J} / \mathrm{mol}$ \\
\hline UA & Heat transfer coefficient & $9.01 \times 10^{5}$ & $\mathrm{~J} /(\min \cdot \mathrm{K})$ \\
\hline$k_{1}$ & Pre-exponential constant of $\mathrm{A} \rightarrow \mathrm{B}$ & $5 \times 10^{4}$ & $\min ^{-1}$ \\
\hline$k_{2}$ & Pre-exponential constant of $\mathrm{A} \rightarrow \mathrm{U}$ & $5 \times 10^{3}$ & $\min ^{-1}$ \\
\hline$k_{3}$ & Pre-exponential constant of $\mathrm{A} \rightarrow \mathrm{R}$ & $5 \times 10^{3}$ & $\min ^{-1}$ \\
\hline$E_{1}$ & Activation energy of $\mathrm{A} \rightarrow \mathrm{B}$ & $5 \times 10^{4}$ & $\mathrm{~J} / \mathrm{mol}$ \\
\hline$E_{2}$ & Activation energy of $\mathrm{A} \rightarrow \mathrm{U}$ & $7.53 \times 10^{4}$ & $\mathrm{~J} / \mathrm{mol}$ \\
\hline$E_{3}$ & Activation energy of $A \rightarrow R$ & $7.53 \times 10^{4}$ & $\mathrm{~J} / \mathrm{mol}$ \\
\hline$R$ & Gas constant & 8.314 & $\mathrm{~J} /(\mathrm{mol} \cdot \mathrm{K})$ \\
\hline$\rho$ & Fluid density in reactor & 1000 & $\mathrm{~g} / \mathrm{L}$ \\
\hline$c_{p}$ & Fluid heat capacity in reactor & 0.231 & $\mathrm{~J} /(\mathrm{g} \cdot \mathrm{K})$ \\
\hline$\rho_{c}$ & Fluid density in cooling jacket & 1000 & $\mathrm{~g} / \mathrm{L}$ \\
\hline$c_{p c}$ & Fluid heat capacity in cooling jacket & 4.2 & $\mathrm{~J} /(\mathrm{g} \cdot \mathrm{K})$ \\
\hline
\end{tabular}

where

$$
\begin{aligned}
& x_{k}=\left[\begin{array}{llll}
C_{\mathrm{A}, \mathrm{k}} & C_{\mathrm{B}, \mathrm{k}} & T_{k} & T_{c, k}
\end{array}\right]^{\top}, u_{k}=\left[\begin{array}{lll}
C_{\mathrm{Af}} & T_{\mathrm{Af}} & T_{\mathrm{cf}, k}
\end{array}\right]^{\top}, \\
& y_{k}=\left[\begin{array}{lll}
C_{\mathrm{B}, \mathrm{k}}^{m} & T_{k}^{m} & T_{c, k}^{m}
\end{array}\right]^{\top} \text {, } \\
& A\left(T_{k}\right)=\left[\begin{array}{cccc}
0.9917-0.1 \sum_{i=1}^{3} g_{i}\left(T_{k}\right) & 0 & 0 & 0 \\
0.1 g_{1}\left(T_{k}\right) & 0.9917 & 0 & 0 \\
\sum_{i=1}^{3}\left(\alpha_{i}+\beta_{1} \delta_{H_{i}}^{0}\right) g_{i}\left(T_{k}\right) & 0 & 0.6017-\beta_{2} \delta_{\mathrm{UA}}^{0} & 0.3900+\beta_{2} \delta_{\mathrm{UA}}^{0} \\
0 & 0 & 0.2145+\beta_{3} \delta_{\mathrm{UA}}^{0} & 0.7355-\beta_{3} \delta_{\mathrm{UA}}^{0}
\end{array}\right], \\
& \alpha_{1}=21.6450, \alpha_{2}=22.5108, \alpha_{3}=23.3766 \text {, } \\
& \beta_{1}=0.0433, \beta_{2}=0.0390, \beta_{3}=0.0214 \text {, } \\
& B=\left[\begin{array}{ccc}
0.0083 & 0 & 0 \\
0 & 0 & 0 \\
0 & 0.0083 & 0 \\
0 & 0 & 0.0500
\end{array}\right], B_{w}=\left[\begin{array}{cccc}
10^{-6} & 0 & 0 & 0 \\
0 & 10^{-6} & 0 & 0 \\
0 & 0 & 10^{-5} & 0 \\
0 & 0 & 0 & 10^{-5}
\end{array}\right] \\
& D_{v}=\left[\begin{array}{ccc}
0.01 & 0 & 0 \\
0 & 0.1 & 0 \\
0 & 0 & 0.1
\end{array}\right] \text {, }
\end{aligned}
$$

${ }_{280} \delta_{H_{i}}^{0}$ and $\delta_{\mathrm{UA}}^{0}$ are scaled from $\delta_{H_{i}}$ and $\delta_{\mathrm{UA}}$ to lie within $[-1,1]$, and $w_{0 k}$ and $v_{0 k}$ follow standard normal distribution to account for discretization errors and 
measurement noises, respectively.

\subsection{Implemented algorithms}

Both the proposed Algorithm 1 and the zonotope-based parity relation in

285

1 , the horizon length $h$ is set to 3 , and the obtained polynomial parity matrix $V\left(T_{k}, \delta^{0}\right)$ is $2 \times 9$ with degree 1 , where $\delta^{0}$ represents $\left[\begin{array}{llll}\delta_{H_{1}}^{0} & \delta_{H_{2}}^{0} & \delta_{H_{3}}^{0} & \delta_{\mathrm{UA}}^{0}\end{array}\right]$. To account for the additive noise $0.1 v_{0 k, T}$ of the measured temperature $T_{k}^{m}$, $T_{k}^{m}-0.1 v_{0 k, T}$ is used to replace $T_{k}$ in the parity matrix $V\left(T_{k}, \delta^{0}\right)$, hence $V\left(T_{k}^{m}-\right.$

$\left.0.1 v_{0 k, T}, \delta^{0}\right)$ is used instead. Then, the uncertainty vector $\boldsymbol{\lambda}$ defined in 22 includes 98 nonzero entries, each of which is a polynomial in terms of $\delta^{0}, w_{0 k}$, and $v_{0 k}$. For the offline GM modeling, 5000 samples $\left\{\boldsymbol{\lambda}^{(i)}\right\}$ of $\boldsymbol{\lambda}$ are generated to construct a sampled data matrix $\boldsymbol{\Lambda}$. To avoid ill-conditioning, dimension reduction via PCA is applied to the sampled data matrix $\boldsymbol{\Lambda}$ such that the

295 data variance in a reduced subspace of dimension 7 can captures $99 \%$ of the total variance. The corresponding PDF of the sampled data in this reduced subspace is approximated by a GM with 2 components. As illustrated in Figure 3. the non-Gaussian distribution of samples in the reduced subspace is well approximated by the obtained GM. The selected confidence level $\gamma$ is $99 \%$. In GM parameters of the residual distribution, and the FD decision is made by following step 8.

As for the zonotope-based method in [15, a zonotope

$$
\mathscr{Z}=\left\{\mathbf{p}+\mathbf{E} \epsilon: \epsilon \in \mathbb{B}^{n_{\epsilon}}\right\}
$$

is constructed offline such that it contains $99 \%$ of the sampled uncertainty vectors $\left\{\boldsymbol{\lambda}^{(i)}\right\}$, where $n_{\epsilon}$ is equal to the rank of the sampled data matrix $\boldsymbol{\Lambda}$, and $\mathbb{B}^{n_{\epsilon}} \in \mathbb{R}^{n_{\epsilon} \times 1}$ is a unitary box composed of $n_{\epsilon}$ unitary intervals $\mathbb{B}=[-1,1]$. In this particular example, the dimensions of $\mathbf{p}, \mathbf{E}$, and $\epsilon$ are $\mathbf{p} \in \mathbb{R}^{98}, \mathbf{E} \in \mathbb{R}^{98 \times 8}$, and $\epsilon \in \mathbb{R}^{8 \times 1}$, respectively. Please refer to [15] for more details of computing the center $\mathbf{p}$ and the shape matrix $\mathbf{E}$ of the zonotope $\mathscr{Z}$. The associated FD decision depends on checking whether the origin lies in the residual confidence zonotope $\left\{\Gamma_{k} \mathbf{p}+\Gamma_{k} \mathbf{E} \epsilon: \epsilon \in \mathbb{B}^{n_{\epsilon}}\right\}$ computed from $(22)$ and (36). This corresponds to the FD logic [15]

$$
\left\{\begin{array}{l}
\nu_{\mathrm{zono}, k} \leq 1 \Rightarrow \text { the monitored system is fault-free } \\
\nu_{\mathrm{zono}, k}>1 \Rightarrow \text { the monitored system is faulty }
\end{array}\right.
$$

where $\nu_{\mathrm{zono}, k}$ is the solution to the linear program

$$
\begin{aligned}
\underset{\epsilon, \nu_{\text {zono }, k}}{\min } & \nu_{\text {zono }, k} \\
\text { s.t. } & \Gamma_{k} \mathbf{p}+\Gamma_{k} \mathbf{E} \epsilon=0 \\
& -\nu_{\text {zono }, k} \leq \epsilon_{i} \leq \nu_{\text {zono }, k}, 1 \leq i \leq n_{\epsilon} \\
& \nu_{\text {zono }, k} \geq 0
\end{aligned}
$$


(a)

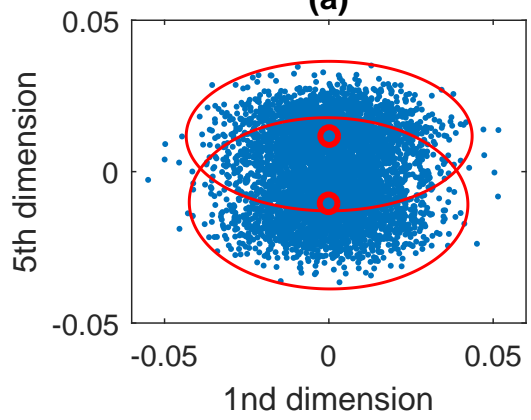

(b)

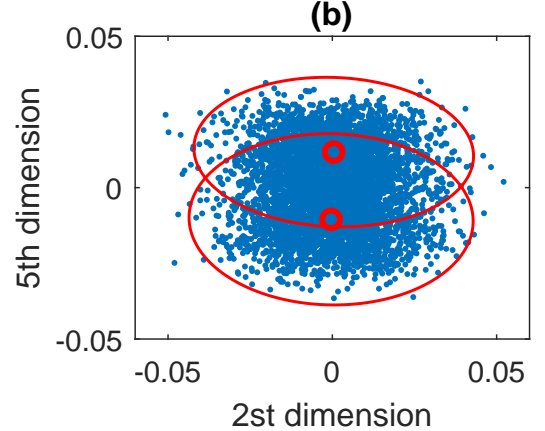

Figure 3: 2-dimensional projections of the sampled $\boldsymbol{\lambda}$ and its GM approximation within the reduced subspace. The blue dots represent the 5000 random samples. Each red circle and ellipse indicate the mean and $99 \%$ confidence set of the corresponding Gaussian component, respectively.

with $\epsilon_{i}$ denoting the $i$ th element of $\epsilon$. Two cases of the solution to (37) imply the detection of faults: (i) a feasible solution with $\nu_{\text {zono, } k}>1$; and (ii) no feasible solution exists. In the latter case, $\nu_{\text {zono, } k}$ can be set to any value larger than 1 for the FD purpose. Note that the zonotope-based method in [15] relies on an LPV residual generator similar to the form of 22 , but does not derive the polynomial parity matrix used in this paper.

\subsection{Performance evaluations and comparisons}

The FD results of one simulation run in the considered two fault scenarios are shown in Figure 6.3. The "residual evaluation" in Figure 6.3 refers to $\nu_{k}$ in (32) and $\nu_{\mathrm{zono}, k}$ in (38). Compared to the zonotope-based method, in both two fault scenarios, our proposed approach is more sensitive to faults, and achieves much fewer number of missed detections after fault injection at $15 \mathrm{~s}$, while giving only one false alarm before fault injection.

In order to evaluate the statistical FD performance, Monte Carlo simulations are performed for $N_{m c}$ times, and the performance metrics are defined by

$$
\begin{aligned}
\mathrm{FAR} & =\frac{\text { total number of false alarms for } t \in[0,15]}{(\text { number of samples for } t \in[0,15]) \times N_{m c}} \times 100 \%, \\
\mathrm{MDR} & =\frac{\text { total number of miss detections for } t \in(15,30]}{(\text { number of samples for } t \in(15,30]) \times N_{m c}} \times 100 \% .
\end{aligned}
$$

The effect of parametric uncertainty bounds on the FD performance is evaluated by the minimal fault magnitude that is detected with an MDR less than $5 \%$ in 100 Monte Carlo runs. As shown in Table 2, when using the tuning parameters described in Section 6.2 as the bound of $\delta_{\mathrm{UA}}$ grows, there is always an increase in the minimal sensor fault magnitude detected with an MDR less than $5 \%$. 

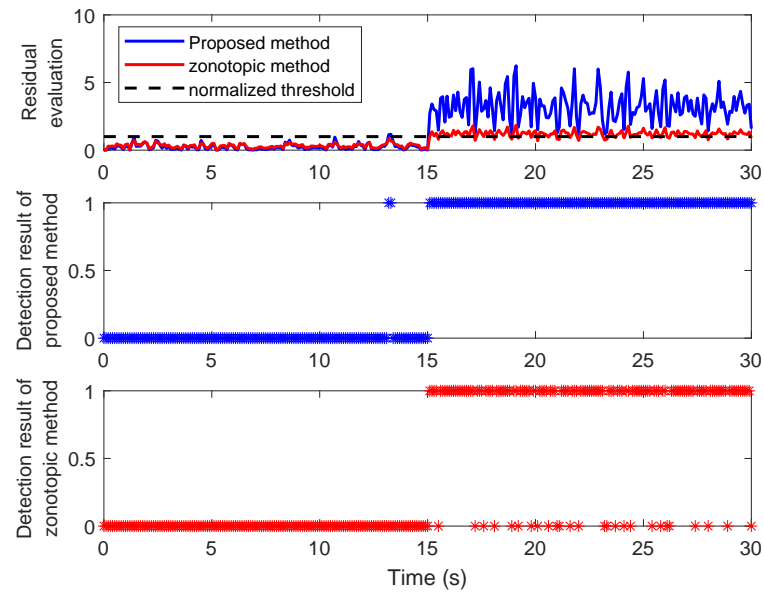

(a) Reactor temperature sensor bias $1.7 \mathrm{~K}$ after $15 \mathrm{~s}$.
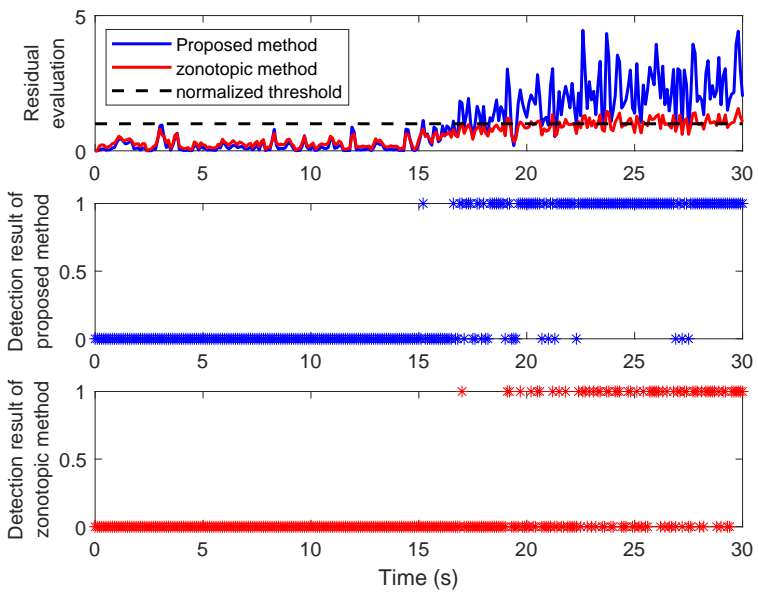

(b) Cooling flow valve stuck at $22 \mathrm{~L} / \mathrm{min}$ after $15 \mathrm{~s}$.

Figure 4: Residual evaluation and detection results of one simulation run in the considered two fault scenarios. In the lower two plots of each subfigure, at each time instant, "1" indicates an alarm from the detector, while "0" represents no reported alarm. 

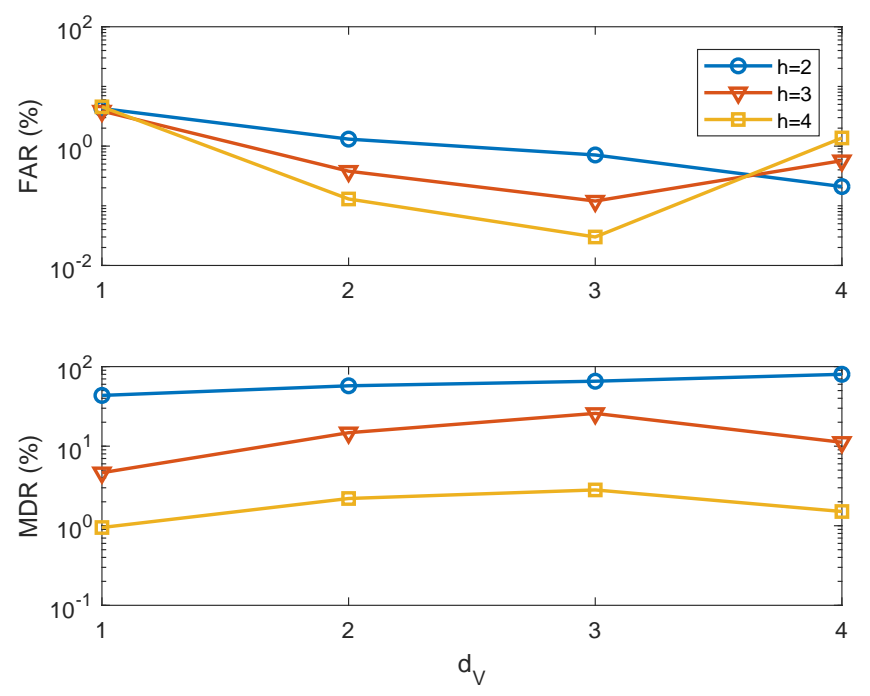

Figure 5: FAR and MDR for different choices of the horizon length $h$ and the degree $d_{V}$. The number of Gaussian components is fixed to 2 . The simulated fault is a constant bias $1.4 \mathrm{~K}$ on the reactor temperature sensor.

How the statistical FD performance depends on tuning parameters is analyzed by performing Monte Carlo runs, and illustrated in Table 3 and Figure 5. It can be seen from Table 3 that

- increasing the reduced dimension $n_{c}$ results in a lower FAR and a higher MDR, due to less information loss in the dimensional reduction step as explained in Remark 4

- by setting a larger confidence level $\gamma$, the achieved FAR decreases while the MDR increases, which is consistent with our expectation.

330 Figure 5 illustrates how the FAR and MDR vary with the horizon length $h$ and the degree $d_{V}$. For each choice of $\left(h, d_{V}\right)$, the dimension reduction step captures $99 \%$ of the total variance of the sampled data matrix of $\boldsymbol{\lambda}$. In Figure 5 , the FAR remains below $5 \%$ in all cases. It is also observed that (i) the MDR becomes higher when $d_{V}$ is larger than its minimal value 1 ; and (ii) for each fixed degree from 1 to 4 , the MDR decreases as $h$ increases.

To show the benefit of exploiting distributional information of uncertain parameters, the PDF of $\delta_{\mathrm{UA}}$ is changed from the truncated Gaussian distribution to the uniform distribution over the same uncertainty range. Compared to case 5 of Table 3 , the same tuning parameters are applied under the uniformly

340 distributed $\delta_{\mathrm{UA}}$, then the obtained FAR $4.17 \%$ almost remains unchanged, and the achieved MDR increases to $5.01 \%$. This is due to not distinguishing high and low probability of occurrence in the uniformly distributed $\delta_{\mathrm{UA}}$, compared to the truncated Gaussian distributed $\delta_{\mathrm{UA}}$. 
Table 2: For different uncertainty ranges of $\delta_{\mathrm{UA}}$, minimal magnitudes of reactor temperature sensor bias that are detected with an MDR less than 5\% in 100 Monte Carlo runs. The tuning parameters of our proposed algorithm are fixed as described in Section 6.2

\begin{tabular}{ccccc}
\hline $\begin{array}{c}\text { Range of } \delta_{\mathrm{UA}} \\
(\mathrm{J} / \mathrm{min} \cdot \mathrm{K})\end{array}$ & $\begin{array}{c}{[-1,1]} \\
\times 10^{4}\end{array}$ & $\begin{array}{c}{[-5,5]} \\
\times 10^{4}\end{array}$ & $\begin{array}{c}{[-9,9]} \\
\times 10^{4}\end{array}$ & $\begin{array}{c}{[-13,13]} \\
\times 10^{4}\end{array}$ \\
\hline Minimal fault magnitude $(\mathrm{K})$ & 1.42 & 1.44 & 1.50 & 1.62 \\
\hline
\end{tabular}

Table 3: FAR and MDR when tuning the reduced dimension $n_{c}$ and the confidence level $\gamma$. The number of Gaussian components is fixed to 2. The simulated fault is a constant bias $1.4 \mathrm{~K}$ on the reactor temperature sensor.

\begin{tabular}{ccccccc}
\hline $\begin{array}{c}\text { Tuning } \\
\text { case }\end{array}$ & $\begin{array}{c}\text { Horizon } \\
\text { length } \\
h\end{array}$ & $\begin{array}{c}\text { Degree } \\
d_{V}\end{array}$ & $\begin{array}{c}\text { Reduced } \\
\text { dimension } \\
n_{c} \text { for } \boldsymbol{\lambda}\end{array}$ & $\begin{array}{c}\text { Confidence } \\
\text { level } \\
\gamma(\%)\end{array}$ & $\begin{array}{c}\text { FAR } \\
(\%)\end{array}$ & $\begin{array}{c}\text { MDR } \\
(\%)\end{array}$ \\
\hline 1 & 3 & 1 & 6 & 95 & 5.56 & 2.41 \\
2 & 3 & 1 & 7 & 95 & 4.15 & 3.03 \\
3 & 3 & 1 & 7 & 99 & 0.97 & 9.19 \\
4 & 3 & 1 & 8 & 99 & 0.86 & 11.67 \\
\hline
\end{tabular}

\section{Conclusions}

This paper presents a probabilistic SM parity relation approach for LPV systems with polynomial dependence on probabilistic parametric uncertainties. A polynomially parameterized parity relation is constructed, and GMs are used to efficiently compute uncertain propagation from stochastic uncertainties to the residual distribution. To achieve an acceptable FAR, the consistency test 350 uses the residual confidence set in the form of a union of ellipsoids. Guidelines for tuning parameters are discussed. The proposed approach is effective for a simulation example, which includes performance comparisons with a zonotopebased method. A comprehensive solution to fault isolation for the considered uncertain LPV system will be a focus of future research.

\section{Acknowledgment}

This work is supported by the National Science Foundation of China, Grant No. 61803163 and No. 61603024. It is also partially supported by the Spanish project DEOCS (ref. MINECO DPI2016-76493).

\section{References}

[1] J. Bokor, Z. Szabó, Fault detection and isolation in nonlinear systems, Annual Reviews in Control 33 (2009) 113-123. 
[2] Y. Yang, S. X. Ding, L. Li, Parameterization of nonlinear observer-based fault detection systems, IEEE Transactions on Automatic Control 61 (2016) 3687-3692.

[3] Y. Wan, T. Keviczky, Real-time fault-tolerant moving horizon air data estimation for the reconfigure benchmark, IEEE Transactions on Control Systems Technology 27 (3) (2019) 997-1011.

[4] H. Hamdi, M. Rodrigues, C. Mechmeche, D. Theilliol, N. B. Braiek, Fault detection and isolation in linear parameter-varying descriptor systems via proportional integral observer, International Journal of Adaptive Control and Signal Processing 26 (3) (2012) 224-240.

[5] S. Armeni, A. Casavola, E. Mosca, Robust fault detection and isolation for LPV systems under a sensitivity constraint, International Journal of Adaptive Control and Signal Processing 23 (1) (2009) 55-72.

[6] K. P. B. Chandra, H. Alwi, C. Edwards, Fault detection in uncertain LPV systems with imperfect scheduling parameter using sliding mode observers, European Journal of Control 34 (2017) 1-15.

[7] L. Chen, R. Patton, P. Goupil, Robust fault estimation using an LPV reference model: ADDSAFE benchmark case study, Control Engineering Practice 49 (2016) 194-203.

[8] D. Henry, J. Cieslak, A. Zolghadri, D. Efimov, $H_{\infty} / H_{-}$LPV solutions for fault detection of aircraft actuator faults: Bridging the gap between theory and practice, International Journal of Robust and Nonlinear Control 25 (5) (2015) 649-672.

[9] M. Rodrigues, H. Hamdi, D. Theilliol, C. Mechmeche, N. BenHadj Braiek, Actuator fault estimation based adaptive polytopic observer for a class of LPV descriptor systems, International Journal of Robust and Nonlinear Control 25 (5) (2015) 673-688.

[10] Z.-H. Zhang, G.-H. Yang, Fault detection for discrete-time uncertain LPV systems using non-minimal order filter, Journal of the Franklin Institute 355 (2) (2018) 902-921.

[11] M. Zhong, Y. Song, S. X. Ding, Parity space-based fault detection for linear discrete time-varying systems with unknown input, Automatica 59 (2015) $120-126$.

[12] R. Lamouchi, T. Raïssi, M. Amairi, M. Aoun, Interval observer framework for fault-tolerant control of linear parameter-varying systems, International Journal of Control 91 (3) (2018) 524-533.

[13] M. M. Seron, J. A. De Doná, Robust fault estimation and compensation for LPV systems under actuator and sensor faults, Automatica 52 (2015) 294-301. 
[14] D. Silvestre, P. Rosa, J. P. Hespanha, C. Silvestre, Fault detection for LPV systems using set-valued observers: A coprime factorization approach, Systems \& Control Letters 106 (2017) 32-39.

[15] J. Blesa, V. Puig, J. Saludes, Robust identification and fault diagnosis based on uncertain multiple input-multiple output linear parameter varying parity equations and zonotopes, Journal of Process Control 22 (2012) 18901912.

[16] S. Ploix, O. Adrot, Parity relations for linear uncertain dynamic systems, Automatic 42 (2006) 1553-1562.

[17] X. Ding, L. Guo, T. Jeinsch, A characterization of parity space and its application to robust fault detection, IEEE Transactions on Automatic Control 44 (2) (1999) 337-343.

[18] P. M. Esfahani, J. Lygeros, A tractable fault detection and isolation approach for nonlinear systems with probabilistic performance, IEEE Transactions on Automatic Control 61 (2016) 633-647.

[19] M. Zhong, L. Zhang, S. X. Ding, D. H. Zhou, A probabilistic approach to robust fault detection for a class of nonlinear systems, IEEE Transactions on Industrial Electronics 64 (2017) 3930-3939.

[20] J. Zhou, Y. Yang, Z. Zhao, S. X. Ding, A fault detection scheme for ship propulsion systems using randomized algorithm techniques, Control Engineering Practice 81 (2018) 65-72.

[21] M. Zhong, Y. Song, T. Xue, R. Yang, W. Li, Parity space-based fault detection by minimum error minimax probability machine, IFAC-PapersOnLine 51 (24) (2018) 1292-1297.

[22] V. Rostampour, R. Ferrari, T. Keviczky, A set based probabilistic approach to threshold design for optimal fault detection, in: Proceedings of American Control Conference, Seattle, WA, 2017, pp. 5422-5429.

[23] C. Combastel, An extended zonotopic and Gaussian Kalman filter (EZGKF) merging set-membership and stochastic paradigms: Toward nonlinear filtering and fault detection, Annual Reviews in Control 42 (2016) 232-243.

[24] M. Pourasghar, C. Combastel, V. Puig, C. Ocampo-Martinez, FD-ZKF: a zonotopic Kalman filter optimizing fault detection rather than state estimation, Journal of Process Control 73 (2019) 89-102.

435

[25] Y. Wan, E. Harinath, R. D. Braatz, Probabilistic robust parity relation for fault detection using polynomial chaos, IFAC-PapersOnLine 50 (1) (2017) 1019-1024. 
[26] Y. Wan, V. Puig, C. Ocampo-Martinez, Y. Wang, R. D. Braatz, Probability-guaranteed set-membership state estimation for polynomially uncertain linear time-invariant systems, in: Proceedings of the 57th IEEE Conference on Decision and Control, Miami Beach, FL, USA, 2018, pp. 2291-2296.

[27] F. Wu, S. Prajna, SOS-based solution approach to polynomial LPV system analysis and synthesis problems, International Journal of Control 78 (8) (2005) 600-611.

[28] E. Süli, D. F. Mayers, An Introduction to Numerical Analysis, Cambridge University Press, 2003.

[29] L. Ljung, System Identification: Theory for the User, Prentice Hall, New Jersey, 1987.

[30] C. Yu, J. Chen, M. Verhaegen, Subspace identification of individual systems in a large-scale heterogeneous network, Automatica 109 (2019) 108517.

[31] C. Yu, L. Ljung, A. Wills, M. Verhaegen, Constrained subspace method for the identification of structured state-space models, IEEE Transactions on Automatic Contro doi:10.1109/TAC.2019.2957703.

[32] G. H. Golub, C. F. Van Loan, Matrix Computations, The Johns Hopkins University Press, 1996.

[33] J. Blesa, V. Puig, J. Saludes, R. M. Fernández-Cantí, Set-membership parity space approach for fault detection in linear uncertain dynamic systems, International Journal of Adaptive Control and Signal Processing 30 (2016) 186-205.

[34] C. G. Baker, K. A. Gallivan, P. Van Dooren, Low-rank incremental methods for computing dominant singular subspaces, Linear Algebra and its Applications 436 (8) (2012) 2866-2888.

[35] C. M. Bishop, Pattern Recognition and Machine Learning, Springer, Singapore, 2006.

[36] C. Bouveyron, C. Brunet-Saumard, Model-based clustering of highdimensional data: A review, Computational Statistics \& Data Analysis 71 (3) (2014) 52-78.

[37] A. Justel, D. Peña, R. Zamar, A multivariate Kolmogorov-Smirnov test of goodness of fit, Statistics \& Probability Letters 35 (3) (1997) 251-259.

[38] P. Mhaskar, J. Liu, P. D. Christofides, Fault-Tolerant Process Control: Methods and Applications, Springer-Verlag, 2013. 